\title{
Homogeneity in the early chemical evolution of the Sextans dwarf spheroidal galaxy ${ }^{\star}$
}

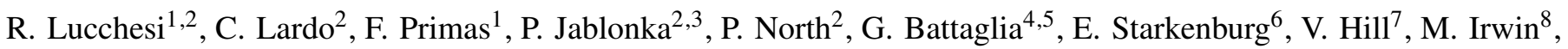 \\ P. Francois ${ }^{3}$, M. Shetrone ${ }^{9}$, E. Tolstoy ${ }^{10}$, and K. Venn ${ }^{11}$
}

${ }^{1}$ European Southern Observatory, Karl-Schwarzschild-str. 2, 85748 Garching bei München, Germany e-mail: romain.lucchesi@epfl.ch

2 Physics Institute, Laboratoire d'astrophysique, École Polytechnique Fédérale de Lausanne (EPFL), Observatoire 1290, Versoix, Switzerland

3 GEPI, Observatoire de Paris, CNRS, Université de Paris Diderot, 92195 Meudon Cedex, France

${ }^{4}$ Instituto de Astrofísica de Canarias (IAC), Calle Via Láctea, s/n, 38205 San Cristóbal de la Laguna, Tenerife, Spain

5 Departamento de Astrofísica, Universidad de La Laguna, 38206 San Cristóbal de la Laguna, Tenerife, Spain

${ }^{6}$ Leibniz-Institut fur Astrophysik Potsdam, An der Sternwarte 16, 14482 Potsdam, Germany

7 Laboratoire Lagrange, Université de Nice Sophia-Antipolis, Observatoire de la Côte d'Azur, France

8 Institute of Astronomy, University of Cambridge, Madingley Road, Cambridge CB3 OHA, UK

9 McDonald Observatory, University of Texas at Austin, Fort David, TX, USA

10 Kapteyn Astronomical Institute, University of Groningen, Landleven 12, 9747 AD Groningen, The Netherlands

11 Department of Physics and Astronomy, University of Victoria, PO Box 3055, STN CSC, Victoria, BC V8W 3P6, Canada

Received 20 January 2020 / Accepted 20 August 2020

\section{ABSTRACT}

\begin{abstract}
We present the high-resolution spectroscopic analysis of two new extremely metal-poor star (EMPS) candidates in the dwarf spheroidal galaxy Sextans. These targets were preselected from medium-resolution spectra centered around the Ca II triplet in the near-infrared and were followed-up at higher resolution with VLT/UVES. We confirm their low metallicities with $[\mathrm{Fe} / \mathrm{H}]=-2.95$ and $[\mathrm{Fe} / \mathrm{H}]=-3.01$, which place them among the most metal-poor stars known in Sextans. The abundances of 18 elements, including $\mathrm{C}$, $\mathrm{Na}$, the $\alpha$, Fe-peak, and neutron-capture elements, are determined. In particular, we present the first unambiguous detection of $\mathrm{Zn}$ in a classical dwarf at extremely low metallicity. Previous indications were made of a large scatter in the abundance ratios of the Sextans stellar population around $[\mathrm{Fe} / \mathrm{H}] \sim-3$ when compared to other galaxies, particularly with very low observed $[\alpha / \mathrm{Fe}]$ ratios. We took the opportunity of reanalyzing the full sample of EMPS in Sextans and find a $[\alpha / \mathrm{Fe}]$ Milky Way-like plateau and a $\sim 0.2$ dex dispersion at fixed metallicity.
\end{abstract}

Key words. stars: abundances - Local Group - galaxies: dwarf - galaxies: formation

\section{Introduction}

In the cosmological $\Lambda$ cold dark matter paradigm $(\Lambda \mathrm{CDM})$, the assembly of large structures in the Universe arose from the coalescence of small systems, and galaxy formation followed the cooling of the primordial gas in dark matter (DM) halos (Press \& Schechter 1974; White \& Rees 1978; Springel et al. 2006). Dwarf spheroidal galaxies (dSphs) are most probably among the best representatives of the protogalactic systems because they are the faintest and most DM-dominated galaxies known in the Universe. However, their exact significance and their role in galaxy formation remain to be clarified. In particular, the abundance patterns in $\mathrm{dSph}$ stars differ drastically from those of the field Milky Way (MW) halo population above $[\mathrm{Fe} / \mathrm{H}] \sim-2$ (Shetrone et al. 2001a; Venn et al. 2004; Tolstoy et al. 2009; Letarte et al. 2010; Jablonka et al. 2015; Hill et al. 2019; Theler et al. 2020). Nonetheless, dwarf galaxies offer the most metal-poor galactic environments that can be investigated. Their stellar populations therefore provide crucial insights into the star formation conditions in the most pristine environments (e.g., Tolstoy et al. 2009; Frebel \& Norris 2015).

\footnotetext{
* Based on UVES observations collected at the ESO, proposal 093.D-
}

Low-mass, long-lived extremely metal-poor (EMP, with $[\mathrm{Fe} / \mathrm{H}] \leq-3$ ) stars have retained the nucleosynthetic signatures of the first generation of stars in their atmospheres. By comparing the chemical patterns of these EMPS in galaxies of very different masses and star formation histories, from ultra-faint and classical dwarfs to the halo of the MW, we can therefore directly test whether the primordial chemical evolution was a universal process and understand the relation between dwarfs and the building blocks of the more massive systems. The proximity of a large number of MW satellites fortunately offers the unique opportunity of studying the relevant aspects of their evolution in great detail and on a star-by-star basis.

The Sextans dSph was discovered by Irwin et al. (1990). At a distance of $\sim 90 \mathrm{kpc}$, it is one of the closest satellites of the MW (Mateo et al. 1995; Lee et al. 2003). It is very extended on the sky with a tidal radius of $120 \pm 20$ arcmin (Cicuéndez et al. 2018) and low surface brightness $\mu_{V, 0}=27.22 \pm 0.08 \mathrm{mag} \cdot \operatorname{arcsec}^{-2}$ (Muñoz et al. 2018). It is a relatively low-mass but strongly dark-matter-dominated classical dSph, $M / L \gg 100$, with a dynamical mass of about $3 \times 10^{8} M_{\odot}$ measured out to a radius of $\sim 3 \mathrm{kpc}$, as seen in Fig. 6 of Breddels \& Helmi (2013; but see also Łokas 2009; Walker et al. 2010; Battaglia et al. 2011 for earlier measurements). The analysis of the color-magnitude 
Table 1. Observation journal.

\begin{tabular}{lccccccc}
\hline \hline ID & $\begin{array}{c}\alpha(J 2000) \\
{[\mathrm{h}: \mathrm{mn}: \mathrm{s}]}\end{array}$ & $\begin{array}{c}\delta(J 2000) \\
{\left[{ }^{\circ}:^{\prime}:{ }^{\prime \prime}\right]}\end{array}$ & {$[\mathrm{Fe} / \mathrm{H}]_{\mathrm{CaT}}$} & $\begin{array}{c}\text { Setting } \\
{[\mathrm{s}]}\end{array}$ & $\begin{array}{c}\lambda \text { range } \\
{[\AA]}\end{array}$ & $\begin{array}{c}S / N \\
{[/ \mathrm{pix}]}\end{array}$ & $\begin{array}{c}\left\langle V_{\text {rad, helio }}\right\rangle \pm \sigma \\
{\left[\mathrm{km} \mathrm{s}^{-1}\right]}\end{array}$ \\
\hline S04-130 & $10: 14: 28.02$ & $-1: 14: 35.80$ & \multirow{2}{*}{-2.89} & Dic1-CD\#2 & $3800-4520$ & 15 & $215.29 \pm 1.11$ \\
& & & & Dic1-CD\#3(Blue) & $4780-5750$ & 45 & $215.64 \pm 0.82$ \\
& & & & Dic1-CD\#3(Red) & $5830-6800$ & 55 & \\
\hline S11-97 & $10: 12: 27.89$ & $-1: 48: 05.20$ & \multirow{2}{*}{2.80} & Dic1-CD\#2 & $3800-4520$ & 16 & $218.06 \pm 1.15$ \\
& & & & Dic1-CD\#3(Blue) & $4780-5750$ & 52 & $218.50 \pm 1.00$ \\
& & & & Dic1-CD\#3(Red) & $5830-6800$ & 59 & \\
\hline
\end{tabular}

Notes. The blue and red parts of the spectra acquired with the 580 red arm CD 3 are considered separately. The $\lambda$ range refers to the spectral ranges used in the analysis.

diagram (CMD) of Sextans reveals a stellar population that is largely dominated by stars older than $\sim 11 \mathrm{Gyr}$ (Lee et al. 2009; Bettinelli et al. 2018), with evidence for radial metallicity and age gradients; the oldest stars forming the most spatially extended component (Lee et al. 2003; Battaglia et al. 2011; Okamoto et al. 2017; Cicuéndez et al. 2018).

Very little is known about the metal-poor tail of the stellar population in Sextans. Only eight EMPS have so far been followed-up at high resolution (Aoki et al. 2009; Tafelmeyer et al. 2010; Honda et al. 2011). The analysis of Aoki et al. (2009) suggested the possible existence of a set of low, subsolar, $[\alpha / \mathrm{Fe}]$ stars and an increased scatter at fixed metallicity compared to the MW or even Sculptor (Starkenburg et al. 2013; Jablonka et al. 2015), which today is the dSph with the largest number of studied EMPS. If confirmed, this has strong implications for the formation processes of Sextans. Cicuéndez \& Battaglia (2018) recently suggested that Sextans could have gone through an accretion or merger episode, which might explain the low $[\alpha / \mathrm{Fe}]$ measurements of Aoki et al. (2009). The most pressing need nevertheless is to increase the number of EMPS with detailed chemical abundances.

The Dwarf Abundances and Radial velocity Team (DART), formed around the ESO Large Program 171.B-0588(A), has surveyed Sextans up to its tidal radius with the mediumresolution grism of FLAMES/GIRAFFE LR8 around the Ca II triplet (CaT). Starkenburg et al. (2010) provided the community with a metallicity calibration based on the CaT valid down to $[\mathrm{Fe} / \mathrm{H}] \sim-4$. This work enabled the identification of a set of new EMP candidates such as in Starkenburg et al. (2013) and Jablonka et al. (2015) and the two targets of this study.

This paper is the first of a series targeting EMP candidates at high resolution in Sextans, Fornax, and Carina to probe the first stages of the chemical enrichment processes occurring in the early Universe. The paper is structured as follows: Sect. 2 presents the observational material and data reduction. The stellar parameters are determined and the elemental abundances are measured in Sect. 3, along with their associated uncertainties. Comments and remarks on the abundances of specific elements are provided in Sect. 4. Finally, we discuss our results and draw conclusions in Sect. 5 and Sect. 6.

\section{Observations and data reduction}

\subsection{Target preselection, observations, and data reduction}

The two EMP candidates of this work, S04-130 and S1197, are red giant branch (RGB) stars that were selected from the CaT sample of Battaglia et al. (2011). The calibration of Starkenburg et al. (2013) led to low-metallicity estimates $[\mathrm{Fe} / \mathrm{H}]_{\mathrm{CaT}}<-2.8$.

S04-130 and S11-97 were followed-up at high resolution with the UVES ${ }^{1}$ spectrograph (Dekker et al. 2000) mounted at the ESO-VLT (program 093.D-0311(B)). We used dichroic1 mode with the gratings 390 blue arm CD 2 centered at $3900 \AA$ and 580 red arm CD 3 centered at $5800 \AA$, together with a $1.2^{\prime \prime}$ slit, leading to a nominal resolution $R \sim 34000$. The total wavelength coverage is $\sim 3200-6800 \AA$, and the effective usable spectral information starts from $\sim 3800 \AA$. Each star has been observed for a total of five hours, split into six individual subexposures. The reduced data, including bias subtraction, flat fielding, wavelength calibration, spectral extraction, and order merging, were taken from the ESO Science Archive Facility.

Table 1 presents some details of the observations (spectral coverage, and signal-to-noise ratios (S/Ns) per spectroscopic pixel) along with the coordinates of stars, their estimated metallicities from the CaT calibration and measured heliocentric radial velocities (see Sect. 2.2). Figure 1 shows the colour-magnitude diagram (CMD) of probable Sextans members from Battaglia et al. (2011). Our UVES targets are highlighted in red. For comparison purposes, we also display the two EMPS, Sex24-72 and Sex11-04, that were observed with UVES and originally presented in Tafelmeyer et al. (2010) and the six EMPS (S10-14, S11-13, S11-37, S12-28, S14-98, and S1519) that were observed with the high-dispersion spectrograph installed on the $8.2 \mathrm{~m}$ Subaru Telescope (Noguchi et al. 2002). They were discussed in Aoki et al. (2009). We refer to the original papers for additional details about the observations. We also show the spatial distribution of these EMPS.

\subsection{Radial velocity measurements and normalization}

The heliocentric radial velocities (RVs) were measured with the IRAF $^{2}$ task rvidlines on each individual exposure. The final $\mathrm{RV}$ is the average of these individual values weighted by their uncertainties. This approach allows us to detect possible binary stars, at least those whose RV variations can be detected within about one year ${ }^{3}$. We did not find any evidence for binarity. After they were corrected for RV shifts, the individual exposures were combined into a single exposure using the IRAF task scombine with sigma clipping. As a final step, each spectrum was

\footnotetext{
1 Ultraviolet and Visual Echelle Spectrograph.

2 Image Reduction and Analysis Facility; Astronomical Source Code Library ascl:9911.002.

3 Observations were performed between 22 April 2015 and 29 January 2016.
} 

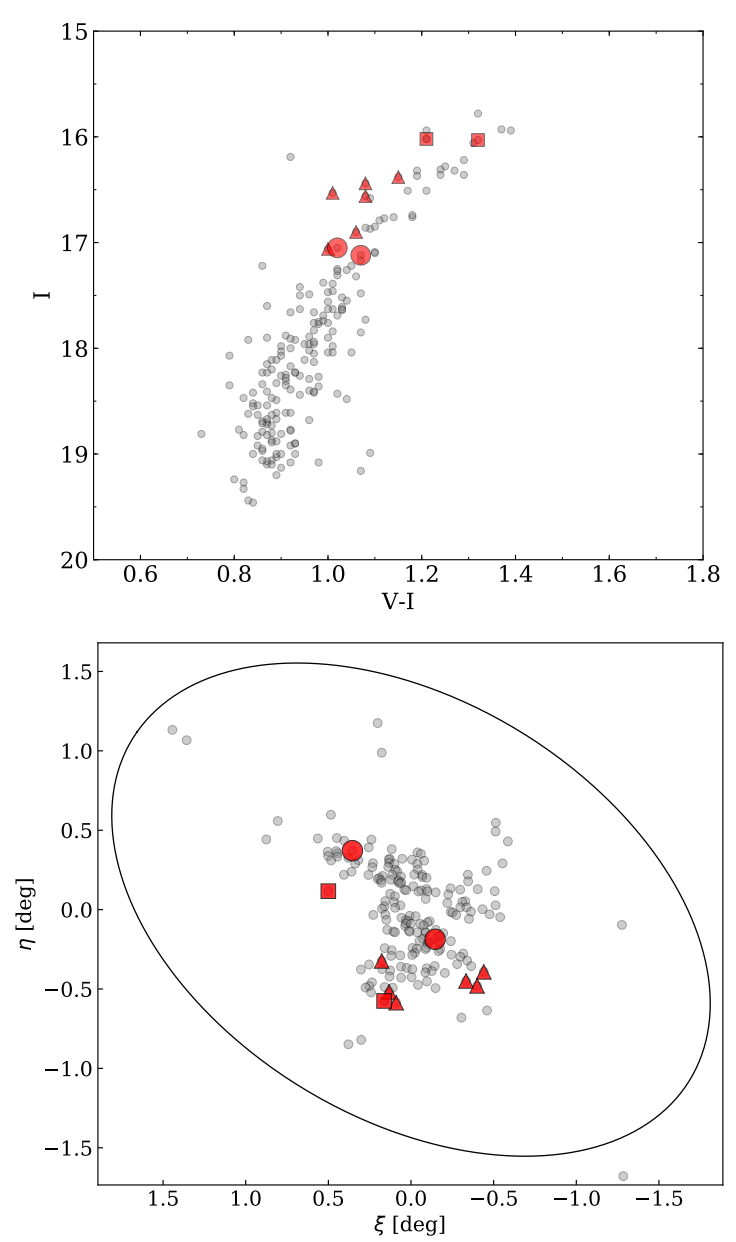

Fig. 1. Top panel: $V-I, I$ CMD of Sextans. Gray circles are probable Sextans members based on their $[\mathrm{Fe} / \mathrm{H}]_{\mathrm{CaT}}$ metallicities and radial velocities (Battaglia et al. 2011). The red symbols show the stars we discuss here. Large circles are the new targets of this work. The samples of Tafelmeyer et al. (2010) and Aoki et al. (2009) are shown with smaller squares and triangles, respectively. Bottom panel: spatial distribution of these stars. The ellipse indicates the tidal radius of Sextans.

visually examined, and the few remaining cosmic rays were removed with the splot routine.

The average RV of each star (Table 1 ) coincides with the RV of Sextans $\left(226.0 \pm 0.6 \mathrm{~km} \mathrm{~s}^{-1}\right)$ within the velocity dispersion $\sigma=8.4 \pm 0.4 \mathrm{~km} \mathrm{~s}^{-1}$ measured by Battaglia et al. (2011). This confirms that our stars are highly probable members.

Spectra were normalized using DAOSPEC (Stetson \& Pancino 2008) for each of the three wavelength ranges presented in Table 1 . We used a 30 to 40 degree polynomial fit.

\section{Chemical analysis}

\subsection{Line list and model atmospheres}

Our line list combines those of Jablonka et al. (2015), Tafelmeyer et al. (2010), and Van der Swaelmen et al. (2013). Information on the spectral lines was taken from the VALD database (Piskunov et al. 1995; Ryabchikova et al. 1997; Kupka et al. 1999, 2000). The central wavelengths and oscillator strengths are given in Table A.1. The adopted solar abundances in Table 3 are from Asplund et al. (2009).

We adopted the new MARCS 1D atmosphere models and selected the Standard composition class, that is, we included the classical $\alpha$-enhancement of +0.4 dex at low metallicity. They were downloaded from the MARCS web site (Gustafsson et al. 2008), and interpolated using Thomas Masseron's interpol_modeles code, which is available on the same web site ${ }^{4}$. Inside a cube of eight reference models, this code performs a linear interpolation on three given parameters: $T_{\text {eff }}, \log g$, and $[\mathrm{Fe} / \mathrm{H}]$.

\subsection{Photometric temperature and gravity}

The atmospheric parameters (APs) were initially determined using photometric information. The first approximated determination of the stellar effective temperature was based on the $V-I, V-J, V-H$, and $V-K$ color indices measured by Battaglia et al. (2011), and $J$ and $K s$ photometry was taken from the VISTA commissioning data, which were also calibrated onto the 2MASS photometric system. We assumed $A v=3.24 \cdot E_{B-V}$ (Cardelli et al. 1989) and $E_{B-V}=0.0477$ (Battaglia et al. 2011) for the reddening correction. The adopted photometric effective temperatures, $T_{\text {eff }}$, are listed in Table 2. They correspond to the simple average of the four color temperatures derived from $V-I, V-J, V-H$, and $V-K$ with the calibration of Ramírez \& Meléndez (2005).

Because only very few Fe II lines can be detected in the very low metallicity regime, we determined the stellar surface gravity $(\log g)$ from their relation with $T_{\text {eff }}$ :

$\log g_{\star}=\log g_{\odot}+\log \frac{M_{\star}}{M_{\odot}}+4 \times \log \frac{T_{\mathrm{eff} \star}}{T_{\mathrm{eff} \odot}}+0.4 \times\left(M_{\mathrm{bol} \star}-M_{\mathrm{bol} \odot}\right)$

assuming $\log g_{\odot}=4.44, T_{\text {eff } \odot}=5790 \mathrm{~K}$, and $M_{\text {bol } \odot}=4.75$ for the Sun. We adopted a stellar mass of $0.8 M_{\odot}$ and calculated the bolometric corrections using the Alonso et al. (1999) calibration and a distance of $d=90 \mathrm{kpc}$ (Karachentsev et al. 2004).

\subsection{Final stellar parameters and abundance determination}

We determined the stellar chemical abundances through the measurement of the equivalent widths (EWs) or the spectral synthesis of atomic transition lines, when necessary. The EWs were measured with DAOSPEC (Stetson \& Pancino 2008). This code performs a Gaussian fit of each individual line and measures its corresponding EW. Although DAOSPEC fits saturated Gaussians to strong lines, it cannot fit the wider Lorentz-like wings of the profile of very strong lines, in particular beyond $200 \mathrm{~m} \AA$. This is especially relevant at very high resolution (Kirby \& Cohen 2012). For some of the strongest lines in our spectra, we therefore derived the abundances by spectral synthesis (see below).

The measured EWs are provided in Table A.1. Values in bracket indicate that the corresponding abundances were derived by spectral synthesis. The abundance derivation from EWs and the spectral synthesis calculation were performed with the Turbospectrum code (Alvarez \& Plez 1998; Plez 2012), which assumes local thermodynamic equilibrium (LTE), but treats continuum scattering in the source function. We used a planeparallel transfer for the line computation; this is consistent with our previous work on EMP stars (Tafelmeyer et al. 2010; Jablonka et al. 2015).

The stellar atmospheric parameters were refined in an iterative manner. In order to constrain $T_{\text {eff }}$ and the microturbulence velocities $\left(v_{\mathrm{t}}\right)$, we required no trend between the abundances

\footnotetext{
4 http://marcs.astro.uu.se
} 
derived from Fe I and excitation potential $\left(\chi \chi_{\text {exc }}\right)$ or the predicted ${ }^{5}$ EWs (Magain 1984). Starting from the initial photometric parameters of Table 2, we adjusted $T_{\text {eff }}$ and $v_{\mathrm{t}}$ by minimizing the slopes of the diagnostic plots allowing the slope to deviate from zero by no more than about twice the uncertainty on the slope. We did not force ionization equilibrium between $\mathrm{Fe} \mathrm{I}$ and Fe II, taking into account that there will likely be nonLTE (NLTE) effects at these low metallicities (Mashonkina et al. 2017a; Ezzeddine et al. 2017). For each iteration the corresponding values of $\log g$ were computed from its relation with $T_{\text {eff }}$, assuming the updated values of $T_{\text {eff }}$, and adjusting the model metallicity to the mean iron abundance derived in the previous iteration.

We excluded from our analysis Fe I lines with $\chi_{\text {exc }}<1.4 \mathrm{eV}$ in order to minimize the NLTE effect on the measured abundances (Jablonka et al. 2015). Additionally, we used only the 580 setting data to calculate $[\mathrm{Fe} / \mathrm{H}]$ and optimize the atmospheric parameters.

We derived the chemical abundances of the strong lines with measured EW > $100 \mathrm{~m} \AA$ by spectral synthesis. These abundances were obtained using our own code, which performs a $\chi^{2}$ minimization between the observed spectral features and a grid of synthetic spectra calculated on the fly with Turbospectrum. A line of a chemical element $X$ is synthesized in a wavelength range of $\sim 50 \AA$. It is optimized by varying its abundance in steps of $0.1 \mathrm{dex}$, from $[X / \mathrm{Fe}]=-1.0$ dex to $[X / \mathrm{Fe}]=+1.0 \mathrm{dex}$. In the same way, the resolution of the synthetic spectra is optimized, starting from the theoretical instrumental resolution, by convolving the spectra in a wide range of Gaussian widths for each abundance step. A second optimization, with abundance steps of $0.01 \mathrm{dex}$, is then performed in a smaller range around the minimum $\chi^{2}$ in order to refine the results. Similarly, the elements with a significant hyperfine structure (HFS) ( $\mathrm{Sc}, \mathrm{Mn}$, $\mathrm{Co}$, and $\mathrm{Ba}$ ) have been determined by running Turbospectrum in its spectral synthesis mode in order to properly take into account blends and the HFS components in the abundance derivation, as in North et al. (2012), Prochaska \& McWilliam (2000) for Sc and Mn, and from the Kurucz web site ${ }^{6}$ for Co and $\mathrm{Ba}$.

The final (spectroscopic) parameters are given in Table 2. The typical uncertainties are $\sim 100 \mathrm{~K}$ on $T_{\text {eff }}, \sim 0.15 \mathrm{dex}$ on $\log g$, assuming a $\pm 0.1 M_{\odot}$ error on $M_{\odot}$ and a 0.2 mag error on $M_{\text {bol }}$, and about $0.15 \mathrm{~km} \mathrm{~s}^{-1}$ on $v_{\mathrm{t}}$.

The final abundances reported in Table 3 are the average abundances from Table A.1 based on EWs or spectral synthesis, weighted by errors. For a few elements (V, Y, and $\mathrm{Zr}$ ) we were only able to place upper limits on their abundances (see Table 3). They are based on visual inspection of the observed spectrum, on which synthetic spectra were overplotted with increasing abundances, until the $\chi^{2}$ deviation became noticeable.

\subsection{Error budget}

1. Uncertainties due to the atmospheric parameters. To estimate the sensitivity of the derived abundances to the adopted atmospheric parameters, we repeated the abundance analysis and varied only one stellar atmospheric parameter at a time by its corresponding uncertainty, keeping the others fixed and repeating

\footnotetext{
5 The use of observed EWs would produce an increase of $v_{\mathrm{t}}$ by $0.1-0.2 \mathrm{~km} \mathrm{~s}^{-1}$, which would be reflected in a decrease of the measured $[\mathrm{Fe} / \mathrm{H}]$ values by a few hundredths of a dex in a systematic way. A variation like this does not change the results in a significant way.

6 http://kurucz.harvard.edu/linelists.html
}

the analysis. The estimated internal errors are $\pm 100 \mathrm{~K}$ in $T_{\text {eff }}$, \pm 0.15 dex in $\log (\mathrm{g})$, and $\pm 0.15 \mathrm{~km} \mathrm{~s}^{-1}$ in $v_{\mathrm{t}}$. Table 4 lists the effects of these changes on the derived abundances for star S04130. With comparable stellar parameters and $\mathrm{S} / \mathrm{N}$, the effects of changes in atmospheric parameters on abundances are expected to be the same for stars S11-97.

2. Uncertainties due to EWs or spectral fitting. The uncertainties on the individual EW measurements $\delta_{\mathrm{EW} i}$ are provided by DAOSPEC (see Table A.1) and computed according to the following formula (Stetson \& Pancino 2008) :

$\delta_{\mathrm{EW} i}=\sqrt{\sum_{p}\left(\delta I_{p}\right)^{2}\left(\frac{\partial \mathrm{EW}}{\partial I_{p}}\right)^{2}+\sum_{p}\left(\delta I_{C_{p}}\right)^{2}\left(\frac{\partial \mathrm{EW}}{\partial I_{C_{p}}}\right)^{2}}$

where $I_{p}$ and $\delta I_{p}$ are the intensity of the observed line profile at pixel $p$ and its uncertainty, and $I_{C_{p}}$ and $\delta I_{C_{p}}$ are the intensity and uncertainty of the corresponding continuum. The uncertainties on the intensities are estimated from the scatter of the residuals that remain after subtraction of the fitted line (or lines, in the case of blends). The corresponding uncertainties $\sigma_{\mathrm{EW} i}$ on individual line abundances are propagated by Turbospectrum. This is a lower limit to the real EW error because systematic errors like the continuum placement are not accounted for. In order to account for additional sources of error, we quadratically added a 5\% error to the EW uncertainty, so that no EW has an error smaller than $5 \%$. This gives a typical uncertainty of $\sigma_{\mathrm{EW}}(\mathrm{Fe} \mathrm{I})=0.08$ rather than 0.04 in Fe I abundance. For the abundances derived by spectral synthesis (e.g., strong lines, hyperfine structure, or carbon from the $G$ band), the uncertainties were visually estimated by gradually changing the parameters of the synthesis until the deviation from the observed line became noticeable.

The final errors listed in Table 3 were computed following the recipes outlined in Hill et al. (2019) and Jablonka et al. (2015). Typical abundance uncertainties for an element $\mathrm{X}$ due to the EW uncertainties $\left(\sigma_{\mathrm{EW} i}\right.$ propagated from $\left.\delta_{\mathrm{EW} i}\right)$ are computed as

$\sigma_{\mathrm{EW}}(X)=\sqrt{\frac{N_{X}}{\sum_{i} 1 / \sigma_{\mathrm{EW} i}^{2}}}$

where $N_{X}$ represents the number of lines measured for element $\mathrm{X}$.

The dispersion $\sigma_{X}$ around the mean abundance of an element $\mathrm{X}$ measured from several lines is computed as

$\sigma_{X}=\sqrt{\frac{\sum_{i}\left(\epsilon_{i}-\bar{\epsilon}\right)^{2}}{N_{X}-1}}$

where $\epsilon$ stands for the logarithmic abundance.

The final error on the elemental abundances is defined as $\sigma_{\text {fin }}=\max \left(\sigma_{\mathrm{EW}}(\mathrm{X}), \sigma_{X} / \sqrt{N_{X}}, \sigma_{\mathrm{Fe}} / \sqrt{N_{X}}\right)$. As a consequence, no element $X$ can have an estimated dispersion $\sigma_{X}<\sigma_{\mathrm{Fe}}$; this is particularly important for species with very few lines.

\section{Specific comments on the abundance determination}

\subsection{Carbon}

The carbon abundance was determined from the intensity of the $\mathrm{CH}$ molecular band between $4323 \AA$ and $4324 \AA$. Some of the carbon is locked in $\mathrm{CO}$ and $\mathrm{CN}$ molecules; as we are not able 
Table 2. Magnitudes, photometric, and spectroscopic parameters.

\begin{tabular}{|c|c|c|c|c|c|c|c|c|c|c|c|c|c|c|c|}
\hline \multirow{3}{*}{ ID } & \multirow{3}{*}{$V$} & \multirow{3}{*}{$I$} & \multirow{3}{*}{$J$} & \multirow{3}{*}{$H$} & \multirow{3}{*}{$K$} & \multicolumn{5}{|c|}{ Photometric parameters } & \multirow[b]{2}{*}{$\log (g)$} & \multicolumn{4}{|c|}{ Final parameters } \\
\hline & & & & & & & & $\overline{T_{\text {eff }}[\mathrm{K}]}$ & & & & $T_{\text {eff }}$ & $\log (g)$ & $v_{\mathrm{t}}$ & {$[\mathrm{Fe} / \mathrm{H}]$} \\
\hline & & & & & & $V-I$ & $V-J$ & $V-H$ & $V-K$ & Mean & [cgs] & {$[\mathrm{K}]$} & [cgs] & {$\left[\mathrm{km} \mathrm{s}^{-1}\right]$} & \\
\hline S04-130 & 18.071 & 17.050 & 16.162 & 15.543 & 15.418 & 4624 & 4735 & 4555 & 4567 & 4620 & 1.13 & 4520 & 1.07 & 1.70 & -2.94 \\
\hline S11-97 & 18.189 & 17.125 & 16.204 & 15.653 & 15.542 & 4543 & 4630 & 4549 & 4567 & 4572 & 1.15 & 4480 & 1.10 & 1.80 & -3.01 \\
\hline
\end{tabular}

Table 3. Derived abundances for S04-130 and S11-97 and the Aoki 2009 stars along with their associated errors (see Sect. 3.4).

\begin{tabular}{|c|c|c|c|c|c|c|c|c|c|c|c|c|c|c|c|c|c|c|c|c|c|}
\hline $\log \epsilon(\mathrm{X})_{\odot}$ & $\begin{array}{l}\mathrm{Fe} \text { I } \\
7.50 \\
\end{array}$ & $\begin{array}{l}\text { Fe II } \\
7.50 \\
\end{array}$ & $\begin{array}{c}\mathrm{C} \\
8.43 \\
\end{array}$ & $\begin{array}{l}\mathrm{Na} \text { I } \\
6.24 \\
\end{array}$ & $\begin{array}{l}\mathrm{Mg} \mathrm{I} \\
7.60\end{array}$ & $\begin{array}{l}\text { Al I } \\
6.45 \\
\end{array}$ & $\begin{array}{r}\mathrm{Si} \mathrm{I} \\
7.51 \\
\end{array}$ & $\begin{array}{l}\mathrm{Ca} \text { I } \\
6.34 \\
\end{array}$ & $\begin{array}{l}\text { Sc II } \\
3.15 \\
\end{array}$ & $\begin{array}{r}\text { Ti I } \\
4.95 \\
\end{array}$ & $\begin{array}{l}\text { Ti II } \\
4.95 \\
\end{array}$ & $\begin{array}{r}\text { V II } \\
3.93 \\
\end{array}$ & $\begin{array}{c}\mathrm{Cr} \mathrm{I} \\
5.64 \\
\end{array}$ & $\begin{array}{l}\mathrm{Mn} \mathrm{I} \\
5.43 \\
\end{array}$ & $\begin{array}{l}\text { Co I } \\
4.99 \\
\end{array}$ & $\begin{array}{l}\mathrm{Ni} \text { I } \\
6.22 \\
\end{array}$ & $\begin{array}{l}\mathrm{Zn} \mathrm{I} \\
4.56 \\
\end{array}$ & $\begin{array}{l}\text { Sr II } \\
2.87 \\
\end{array}$ & $\begin{array}{r}\text { Y II } \\
2.21 \\
\end{array}$ & $\begin{array}{l}\mathrm{Zr} \text { II } \\
2.58 \\
\end{array}$ & $\begin{array}{l}\text { Ba II } \\
2.18 \\
\end{array}$ \\
\hline \multicolumn{22}{|l|}{ S04-130 } \\
\hline Nb lines* & 42 & 5 & 1 & 2 & 5 & 2 & - & 9 & 7 & 11 & 19 & 1 & 7 & 5 & 4 & 4 & 1 & 2 & 2 & 1 & 4 \\
\hline $\log \epsilon(\mathrm{X})$ & 4.56 & 4.80 & 4.96 & 3.80 & 5.11 & 2.96 & - & 3.58 & 0.37 & 2.04 & 2.30 & $<0.95$ & 2.53 & 2.21 & 2.05 & 3.40 & 2.25 & 0.06 & $<-1.16$ & $<-0.25$ & -1.56 \\
\hline$[\mathrm{X} / \mathrm{H}]$ & -2.94 & -2.70 & -3.47 & -2.44 & -2.49 & -3.49 & - & -2.76 & -2.78 & -2.91 & -2.65 & $<-2.98$ & -3.11 & -3.22 & -2.94 & -2.82 & -2.31 & -2.80 & $<-3.37$ & $<-2.83$ & -3.74 \\
\hline$[\mathrm{X} / \mathrm{Fe}]$ & - & +0.24 & -0.53 & +0.50 & +0.45 & -0.55 & - & +0.18 & +0.16 & +0.03 & +0.29 & $<-0.04$ & -0.17 & -0.28 & -0.00 & +0.11 & +0.63 & +0.13 & $<-0.43$ & $<+0.11$ & -0.80 \\
\hline Error & 0.11 & 0.11 & 0.15 & 0.11 & 0.11 & 0.11 & - & 0.14 & 0.15 & 0.11 & 0.15 & - & 0.11 & 0.11 & 0.13 & 0.17 & 0.11 & 0.11 & - & - & 0.11 \\
\hline $\mathrm{Nb}$ lines* & 44 & 4 & 1 & 2 & 5 & 1 & - & 11 & 7 & 10 & 20 & - & 9 & 5 & 4 & 4 & 1 & 2 & 2 & 1 & 3 \\
\hline $\log \epsilon(\mathrm{X})$ & 4.49 & 4.54 & 4.87 & 3.79 & 5.08 & 3.05 & - & 3.59 & 0.37 & 1.97 & 2.26 & - & 2.52 & 2.07 & 1.78 & 3.37 & 2.10 & -0.48 & $<-1.17$ & $<-0.39$ & -1.61 \\
\hline$[\mathrm{X} / \mathrm{H}]$ & -3.01 & -2.96 & -3.56 & -2.45 & -2.52 & -3.40 & - & -2.75 & -2.78 & -2.98 & -2.69 & - & -3.12 & -3.36 & -3.21 & -2.85 & -2.46 & -3.34 & $<-3.38$ & $<-2.97$ & -3.79 \\
\hline [X/Fe] & - & +0.05 & -0.55 & +0.56 & +0.49 & -0.39 & - & +0.26 & +0.23 & +0.03 & +0.32 & - & -0.11 & -0.35 & -0.20 & +0.16 & +0.55 & -0.34 & $<-0.37$ & $<+0.04$ & -0.78 \\
\hline Error & 0.11 & 0.27 & 0.16 & 0.11 & 0.11 & 0.11 & - & 0.11 & 0.13 & 0.11 & 0.13 & - & 0.16 & 0.11 & 0.11 & 0.18 & 0.11 & 0.18 & - & - & 0.11 \\
\hline \multicolumn{22}{|l|}{ S10-14 } \\
\hline $\mathrm{Nb}$ lines* & 30 & 4 & - & - & 1 & - & - & 1 & - & - & 2 & - & 2 & - & - & - & - & - & - & - & 1 \\
\hline $\log \epsilon(\mathrm{X})$ & 4.49 & 4.63 & - & - & 4.88 & - & - & 3.55 & - & - & 2.12 & - & 2.20 & - & - & - & - & - & - & - & -1.72 \\
\hline$[\mathrm{X} / \mathrm{H}]$ & -3.01 & -2.87 & - & - & -2.72 & - & - & -2.79 & - & - & -2.83 & - & -3.44 & - & - & - & - & - & - & - & -3.90 \\
\hline Error & 0.19 & 0.38 & - & - & 0.20 & - & - & 0.20 & - & - & 0.18 & - & 0.38 & - & - & - & - & - & - & - & 0.18 \\
\hline \multicolumn{22}{|l|}{ S11-13 } \\
\hline $\mathrm{Nb}$ lines* & 25 & 2 & - & - & 1 & - & - & 2 & 1 & 1 & 1 & - & 2 & - & - & 1 & - & - & - & - & 2 \\
\hline $\log \epsilon(\mathrm{X})$ & 4.45 & 4.69 & - & - & 4.78 & - & - & 3.47 & 0.16 & 1.64 & 2.38 & - & 2.10 & - & - & 3.36 & - & - & - & - & -1.65 \\
\hline$[\mathrm{X} / \mathrm{H}]$ & -3.05 & -2.81 & - & - & -2.82 & - & - & -2.87 & -2.99 & -3.31 & -2.58 & - & -3.53 & - & - & -2.86 & - & - & - & - & -3.83 \\
\hline$[\mathrm{X} / \mathrm{Fe}]$ & - & +0.24 & - & - & +0.23 & - & - & +0.18 & +0.06 & -0.26 & +0.48 & - & -0.48 & - & - & +0.19 & - & - & - & - & -0.78 \\
\hline Error & 0.20 & 0.20 & - & - & 0.20 & - & - & 0.20 & 0.20 & 0.20 & 0.20 & - & 0.20 & - & - & 0.20 & - & - & - & - & 0.20 \\
\hline \multicolumn{22}{|l|}{ S11-37 } \\
\hline $\mathrm{Nb}$ lines* & 26 & 3 & - & - & 1 & - & - & 2 & 1 & 1 & 2 & - & 2 & - & - & 1 & - & - & - & - & 2 \\
\hline $\log \epsilon(X)$ & 4.52 & 4.70 & - & - & 4.94 & - & - & 3.51 & 0.31 & 1.76 & 2.17 & - & 2.21 & - & - & 3.30 & - & - & - & - & -1.68 \\
\hline$[\mathrm{X} / \mathrm{H}]$ & -2.98 & -2.80 & - & - & -2.66 & - & - & -2.83 & -2.84 & -3.19 & -2.78 & - & -3.43 & - & - & -2.92 & - & - & - & - & -3.86 \\
\hline$[\mathrm{X} / \mathrm{Fe}]$ & - & +0.18 & - & - & +0.32 & - & - & +0.15 & +0.14 & -0.21 & +0.20 & - & -0.45 & - & - & +0.06 & - & - & - & - & -0.87 \\
\hline Error & 0.18 & 0.18 & - & - & 0.20 & - & - & 0.16 & 0.20 & 0.20 & 0.69 & - & 0.19 & - & - & 0.19 & - & - & - & - & 0.30 \\
\hline \multicolumn{22}{|l|}{ S12-28 } \\
\hline $\mathrm{Nb}$ lines* & 35 & 5 & - & - & 2 & - & - & 3 & 3 & 2 & 5 & - & 1 & 1 & - & - & - & - & - & - & 2 \\
\hline $\log \epsilon(X)$ & 4.50 & 4.59 & - & - & 4.96 & - & - & 3.61 & 0.11 & 1.89 & 2.29 & - & 2.39 & 2.16 & - & - & - & - & - & - & -1.06 \\
\hline$[\mathrm{X} / \mathrm{H}]$ & -3.00 & -2.91 & - & - & -2.64 & - & - & -2.73 & -3.04 & -3.06 & -2.66 & - & -3.25 & -3.27 & - & - & - & - & - & - & -3.24 \\
\hline$[\mathrm{X} / \mathrm{Fe}]$ & - & +0.09 & - & - & +0.36 & - & - & +0.27 & -0.04 & -0.06 & +0.34 & - & -0.25 & -0.27 & - & - & - & - & - & - & -0.24 \\
\hline $\mathrm{Nb}$ lines* & 17 & 1 & - & - & 1 & - & - & 3 & 1 & 1 & 4 & - & 2 & - & - & - & - & - & - & - & 2 \\
\hline $\log \epsilon(X)$ & 4.58 & 5.07 & - & - & 4.96 & - & - & 3.89 & 0.21 & 2.57 & 2.53 & - & 2.38 & - & - & - & - & - & - & - & -1.63 \\
\hline$[\mathrm{X} / \mathrm{H}]$ & -2.92 & -2.43 & - & - & -2.64 & - & - & -2.45 & -2.94 & -2.38 & -2.42 & - & -3.26 & - & - & - & - & - & - & - & -3.81 \\
\hline$[\mathrm{X} / \mathrm{Fe}]$ & - & +0.49 & - & - & +0.29 & - & - & +0.47 & -0.01 & +0.54 & +0.50 & - & -0.33 & - & - & - & - & - & - & - & -0.89 \\
\hline Error & 0.17 & 0.20 & - & - & 0.20 & - & - & 0.23 & 0.21 & 0.20 & 0.48 & - & 0.18 & - & - & - & - & - & - & - & 0.31 \\
\hline \multicolumn{22}{|l|}{ S15-19 } \\
\hline $\mathrm{Nb}$ lines* & 22 & 3 & - & - & 2 & - & - & 5 & 1 & 1 & 9 & - & 2 & - & - & 1 & - & 1 & - & - & 2 \\
\hline $\log \epsilon(\mathrm{X})$ & 4.28 & 4.19 & - & - & 5.01 & - & - & 3.64 & 0.64 & 2.05 & 1.94 & - & 2.30 & - & - & 2.96 & - & -1.27 & - & - & -0.30 \\
\hline$[\mathrm{X} / \mathrm{H}]$ & -3.22 & -3.31 & - & - & -2.59 & - & - & -2.70 & -2.51 & -2.90 & -3.01 & - & -3.34 & - & - & -3.26 & - & -4.14 & - & - & -2.48 \\
\hline$[\mathrm{X} / \mathrm{Fe}]$ & - & -0.09 & - & - & +0.63 & - & - & +0.52 & +0.71 & +0.32 & +0.21 & - & -0.12 & - & - & -0.04 & - & -0.92 & - & - & +0.74 \\
\hline Error & 0.19 & 0.23 & - & - & 0.19 & - & - & 0.19 & 0.21 & 0.19 & 0.21 & - & 0.19 & - & - & 0.19 & - & 0.28 & - & - & 0.19 \\
\hline
\end{tabular}

Notes. *Number of lines kept after a careful selection of the best fitted lines.

to measure the oxygen and nitrogen abundances, we assumed that $[\mathrm{O} / \mathrm{Fe}]=[\mathrm{Mg} / \mathrm{Fe}]$ and that $[\mathrm{N} / \mathrm{Fe}]$ has a solar value, following Tafelmeyer et al. (2010) and Starkenburg et al. (2013). Synthetic spectra were then compared to the observed spectra. As an example, Fig. 2 shows the comparison between the observed spectrum of S04-130 and five synthetic spectra computed with increasing carbon abundances.

\section{2. $\alpha$ elements}

Magnesium. The Mg abundance is based on five lines that are distributed from the violet to the yellow part of the spectrum. Four of them are rather strong, with EW > $100 \mathrm{m \AA}$ and non-Gaussian line profiles. The abundances of these lines are not consistent with the weaker line. 
Table 4. Changes in the mean abundances $\Delta[\mathrm{X} / \mathrm{H}]$ caused by a $\pm 100 \mathrm{~K}$ change in $T_{\text {eff }}, \mathrm{a} \pm 0.15$ dex change in $\log (\mathrm{g})$ and a $\pm 0.15 \mathrm{~km} \mathrm{~s}^{-1}$ change on $v_{\mathrm{t}}$ for star S04-130.

\begin{tabular}{|c|c|c|c|c|c|c|}
\hline \multirow[b]{2}{*}{$X$} & \multicolumn{6}{|c|}{$\delta \log \epsilon(\mathrm{X})$} \\
\hline & $+\Delta T_{\mathrm{eff}}$ & $+\Delta \log (g)$ & $+\Delta v_{\mathrm{t}}$ & $-\Delta T_{\mathrm{eff}}$ & $-\Delta \log (g)$ & $-\Delta v_{\mathrm{t}}$ \\
\hline $\mathrm{Fe} \mathrm{I}$ & + & -0.01 & 0.03 & -0 & +0.00 & $0 \Omega$ \\
\hline Fe II & & & -0.03 & & & \\
\hline $\mathrm{Na} \mathrm{I}$ & +0.14 & -0.01 & -0.07 & -0 & & \\
\hline$M g_{I}$ & +0.07 & -0.01 & -0.03 & -0.08 & +( & +0 \\
\hline Al I & +0.17 & -0 & -0.09 & -0 & & \\
\hline$I$ & & & -0 & -0 & & \\
\hline Sc II & +0.05 & +0 . & -0.02 & -0 & & +0.02 \\
\hline Ti I & +0.16 & -0.01 & -0.02 & -0 . & & +0.01 \\
\hline & +0.03 & +0 . & -0.03 & -0 . & & +0.03 \\
\hline Cr I & +0 . & 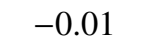 & -0.02 & -0 & 1 & +0.02 \\
\hline Mn I & +0.13 & -0.01 & -0.04 & -0.13 & & +0.02 \\
\hline$C_{0}$ & +0.19 & +0.00 & -0.11 & -0 & +0.00 & +0.12 \\
\hline & & & -0 . & -0 . & & \\
\hline $\mathrm{Zn} I$ & +0.03 & +0.02 & -0.02 & -0.02 & -0.03 & +0.01 \\
\hline Sr II & +0.12 & +0.02 & -0.17 & -0.12 & -0.04 & +0.12 \\
\hline Ba II & +0.07 & +0.05 & -0.02 & -0.06 & -0.05 & +0.02 \\
\hline
\end{tabular}

For this reason, we decided to derive the $\mathrm{Mg}$ abundance through spectral synthesis, after which all lines had consistent abundances. The EW-based abundances derived for the weaker line are consistent with those obtained using spectral synthesis. This confirms the validity of this method. One more $\mathrm{Mg}$ I line is present in our spectra, at $\lambda 4351 \AA$, but it was discarded because it is strongly blended with $\mathrm{Fe}, \mathrm{CH}$, and $\mathrm{Cr}$ I lines.

Silicon. Two Si lines are detected in our spectra, but they are in a noisy part of the spectrum and fall very close to the strong Ca II absorption bands. The continuum level is hard to determine in this region, and the derived abundances strongly depend on it. Therefore we did not derive any silicon abundance.

Titanium. The Ti I abundances rely on 10-11 faint lines, all giving consistent abundance values. The Ti II abundances are based on 19-20 lines. They are slightly more scattered as many of them are rather strong. The mean abundances of Ti I and Ti II are different by $\Delta$ (Ti II-Ti I $)=+0.26$ to +0.29 dex. This is explained by the fact that Ti II is less sensitive to NLTE effects than its neutral state. Thus, following Jablonka et al. (2015), for the purpose of our discussion we adopted the Ti II abundances as the most representative of the titanium content in our stars.

\subsection{Iron-peak elements}

Scandium. The Sc abundance is based on seven lines. They are all derived by spectral synthesis taking into account their HFS components. The smallest line ( $25 \mathrm{~m} \AA$ ) and the bluest line $(\lambda 4246.8 \AA$ ) both give slightly larger abundances, and the other four lines are more consistent.

Chromium. $\mathrm{Cr}$ relies on seven to nine lines. Four are rather strong (EW $>80 \mathrm{m \AA})$, and the other five are weaker $(\mathrm{EW}<50 \mathrm{~m} \AA)$. Strong and weaker lines give more consistent results when the abundances are determined through spectral synthesis. The $\lambda 5208 \AA$ line is blended with an Fe I line and therefore had to be analyzed through spectral synthesis.

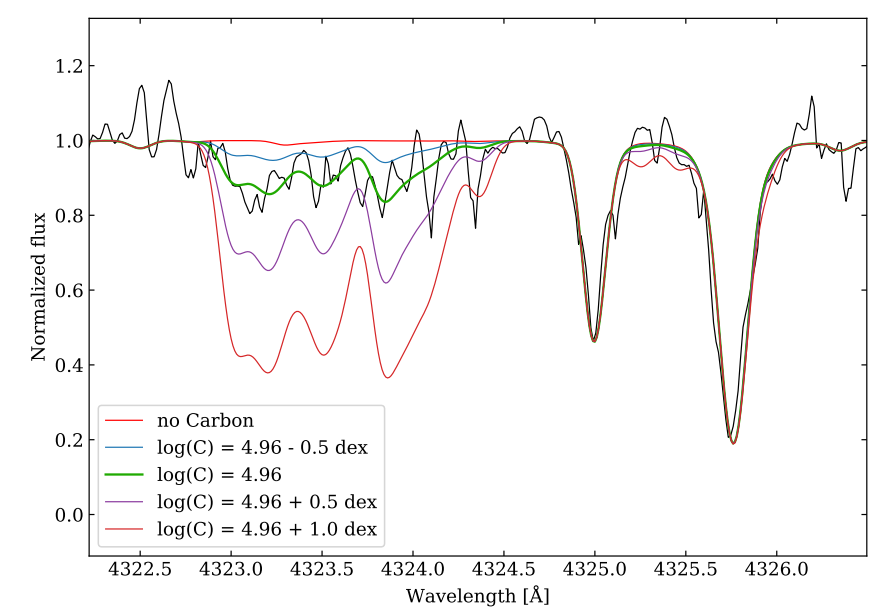

Fig. 2. From top to bottom, examples of synthetic spectra in the $\mathrm{CH}$ band are computed with increasing carbon abundances and are overplotted on the observed spectrum of S04-130 (black). The third green line shows our best representation of the data.

Manganese. All Mn lines (five) were synthesized taking into account their HFS components. They give consistent abundance results.

Cobalt. Four lines are present in our spectra. They are all affected by hyperfine structure, and two of them ( $\lambda 3894 \AA$ and $\lambda 3995 \AA$ ) are blended with Fe I lines. Therefore we derived all four line abundances by spectral synthesis.

Nickel. The Ni abundance is estimated from one or two strong lines and several very faint ones. Spectral synthesis gives consistent abundances for all lines.

Zinc. Only one line of zinc is present in our observed spectra, at $4810 \AA$. The detection is clear but the line is faint, therefore the zinc abundance was derived through spectral synthesis.

\subsection{Neutron-capture elements}

Strontium. Two strong lines of strontium are detected in the blue part of our UVES spectra, but the abundances derived from their EWs are quite discrepant $(0.2$ dex and 0.8 dex in our two stars, respectively). The $4215.5 \AA$ line of the star S11-97 is affected by the $\mathrm{CN}$ molecular band in this region. Spectral synthesis taking into account the carbon abundance derived in the $\mathrm{CH}$ band led to an abundance that agrees better with the $4077.7 \AA$ A line.

Yttrium. Two very faint lines $(<15 \mathrm{~m} \AA)$ of yttrium were detected in our spectra, but we were only able to place upper limits on the Y abundance in our stars.

Barium. Four lines of barium are present in our wavelength ranges. One is very faint $(\lambda 5853 \AA)$ and detected for only one star, and the other three lines are strong. Two of them are blended with weak iron lines $(\lambda 4934 \AA$ and $\lambda 6141 \AA$ ). Therefore we proceeded by spectral synthesis, taking into account all blends and the Ba HFS components. Barium has five isotopes; different fractions of even- $\mathrm{A}$ and odd- $\mathrm{A}$ ( $\mathrm{A}=$ atomic mass) nuclei $\left({ }^{134} \mathrm{Ba}+{ }^{136} \mathrm{Ba}+{ }^{138} \mathrm{Ba}\right):\left({ }^{135} \mathrm{Ba}+{ }^{137} \mathrm{Ba}\right)$ were tested: the $82: 18$ solar fraction, and the $r$-process fractions of $54: 46$ and 28:72. The Ba $\lambda 4934 \AA$ resonance line is more sensitive than the three subordinate lines to the adopted fraction. The solar $82: 18$ fraction led to the best agreement between the resonance 
and the subordinates lines. We refer to Jablonka et al. (2015), Mashonkina et al. (2017b) for a more detailed investigation of the possible cause.

\section{Discussion}

\subsection{Carbon}

Figure 3 shows that none of our stars can be considered as carbon-enhanced based on the Aoki et al. (2007) criterion. Nonetheless, our stars are evolved enough to have converted $\mathrm{C}$ into $\mathrm{N}$ by the CNO cycle, as they are above $\log \left(L_{\star} / L_{\odot}\right)=2.3$, that is, the limit above which a metal-poor $0.8 M_{\odot}$ star is thought to undergo additional mixing between the bottom of the stellar convective envelope and the outer layer of the advancing hydrogen shell (see Placco et al. 2014, and references therein for a discussion).

Placco et al. (2014) developed a procedure for correcting the measured carbon abundances based on stellar model evolution and depending on the $\log (\mathrm{g})$ of the stars. They showed that when these corrections were applied to their dataset, the fraction of carbon-rich stars $[\mathrm{C} / \mathrm{Fe}]>+0.7$ increased to $43 \%$ for $[\mathrm{Fe} / \mathrm{H}]<-3$. The corrections are interpolated ${ }^{7}$ at given $\log (\mathrm{g})$, $[\mathrm{Fe} / \mathrm{H}]$ and $[\mathrm{C} / \mathrm{Fe}]$. For the star S04-130, the corresponding correction is +0.73 dex, resulting in a ratio of $[\mathrm{C} / \mathrm{Fe}]=0.20 \mathrm{dex}$. For S11-97 the derived correction is +0.74 dex, resulting in $[\mathrm{C} / \mathrm{Fe}]=0.19 \mathrm{dex}$. This retains the two stars immediately below the limit of C-rich stars defined by Aoki et al. (2007) (Fig. 3, empty circles).

In the MW halo a significant fraction of metal-poor stars, that is, stars with $[\mathrm{Fe} / \mathrm{H}] \leq-2$, is enriched in carbon $([\mathrm{C} / \mathrm{Fe}]>0.7$ $\operatorname{dex})^{8}$. The fraction of carbon-enriched metal-poor (CEMP) stars appears to be a function of decreasing metallicity (e.g., Beers \& Christlieb 2005). This suggests that large amounts of carbon were synthesized in the early Universe when the oldest and most metal-poor stars formed.

Despite extensive observational searches, only a few carbonrich stars have been known in dSphs until very recently, even at low metallicities. In Sextans, one CEMP star has been identified with $[\mathrm{C} / \mathrm{Fe}]=+1$ by Honda et al. (2011) (star S15-19 from Aoki et al. 2009), and one moderately enhanced carbon star with $[\mathrm{C} / \mathrm{Fe}]=+0.4$ by Tafelmeyer et al. (2010). A CEMP star has been also discovered in Draco (Cohen \& Huang 2009) and Sculptor (Skúladóttir et al. 2015; Salvadori et al. 2015). Finally, Kirby et al. (2015) studied a sample of 398 giants in Sculptor, Fornax, Ursa Minor, and Draco. They identified 11 very carbonrich giants (eight were previously known) in three dSphs (Fornax, Ursa Minor, and Draco).

Because the MW halo is expected to be at least partially composed of disrupted dSphs accreted by the Galactic halo, it is important to carefully compare the carbon-enhanced fraction of the MW stellar halo with the values observed in dSphs. The recent study of Chiti et al. (2018) at low resolution $(R \sim 2000)$ found that CEMP stars at metallicities below $[\mathrm{Fe} / \mathrm{H}]<-3.0$ constitute $36 \%$ of the observed stars in Sculptor. The measured fraction is comparable to the fraction of $30 \%$ observed by Yong et al. (2013) in the MW halo (Placco et al. 2014), suggesting that some stars that now populating the Galactic halo may have originated from accreted early analogs of dwarf galaxies. More and higher resolution studies are needed to confirm these fractions inside the dwarf galaxies. Moreover, the identification of carbon-rich

\footnotetext{
7 https://vplacco.pythonanywhere.com/

8 Throughout this paper, we adopt the Aoki et al. (2007) criterion to define carbon-enhanced objects.
}

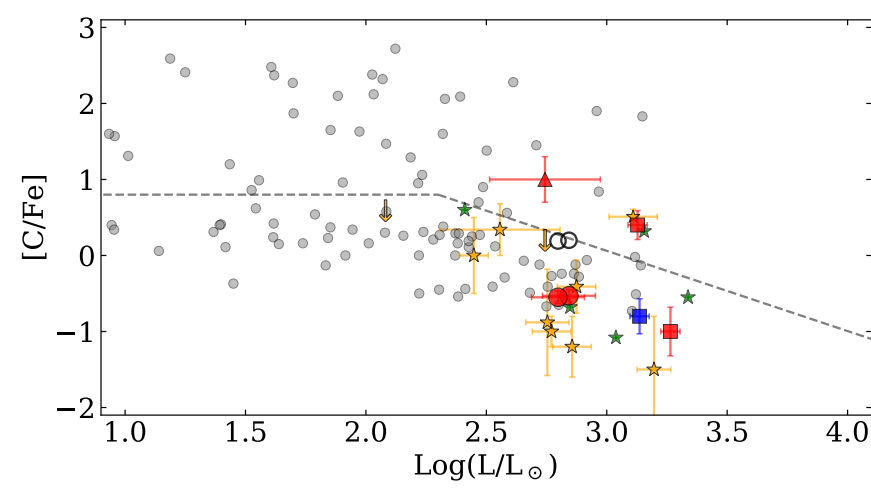

Fig. 3. $[\mathrm{C} / \mathrm{Fe}]$ as a function of $\log \left(L / L_{\odot}\right)$ for Galactic dwarf satellite and halo red giants with metallicities $[\mathrm{Fe} / \mathrm{H}]<-2.5$. The Sextans stars we analyzed are represented by large red circles. Red squares are Sextans stars from Tafelmeyer et al. (2010), the red triangle is the Sextans carbon-rich star from Honda et al. (2011). Gray dots denote the $[\mathrm{C} / \mathrm{Fe}]$ abundances of MW halo stars from Yong et al. (2013). RGB stars in Sculptor (Jablonka et al. 2015; Simon et al. 2015; Tafelmeyer et al. 2010), Fornax (Tafelmeyer et al. 2010), and Draco (Shetrone et al. 2013; Cohen \& Huang 2009) are shown in orange, blue, and green; respectively. The dotted line is the Aoki et al. (2007) dividing line for carbon enhancement, which takes into account the depletion of carbon with evolution along the RGB.

stars and comparisons between galaxies may well be revised in light of 3D NLTE treatment at similar stellar evolutionary stage. Amarsi et al. (2019) have shown that for main-sequence stars, the rise in carbon overabundance with decreasing metallicity vanishes. However, most of our knowledge in dwarf galaxies comes from giant stars, therefore the effect of 3D NLTE on C still remains to be uncovered.

\subsection{Sodium}

Figure 4 presents the results of LTE calculations for $[\mathrm{Na} / \mathrm{Fe}]$ ratios as a function of metallicity in Sextans (this paper and Tafelmeyer et al. 2010), Sculptor (Jablonka et al. 2015), and Fornax (Tafelmeyer et al. 2010), compared to [Na/Fe] abundances measured in MW halo stars. Similarly to the other dwarfs, Sextans follows the MW trend. Our stars lie on the upper envelope of the dispersion range. We did not consider the $\mathrm{Na}$ abundances measured by Aoki et al. (2009) because they were obtained from EW measurements of two strong Na D features at 5889 and $5895 \AA$ with an EW that typically exceeds $100 \mathrm{~m} \AA$ (see Sect. 5.3). However, the Na doublet at 5889 and $5895 \AA$ is also strongly affected by NLTE. According to the NLTE calculation by Lind et al. $(2011)^{9}$, the NLTE corrections for the two Na lines are both negative.

Mashonkina et al. (2017b) computed NLTE corrections for 59 very metal-poor stars in seven dSphs and the MW halo. At metallicity $[\mathrm{Fe} / \mathrm{H}]=-3$, the $\mathrm{Na} \Delta[\mathrm{NLTE}-\mathrm{LTE}]$ range from -0.2 to -0.4 dex, which seems to agree with the Lind et al. (2011) computations. These order-of-magnitude corrections for the NLTE are mentioned to provide an idea of where the stars might stand.

\section{3. $\alpha$ elements}

The plateau at $[\alpha / \mathrm{Fe}] \sim+0.4$ dex seen in the MW metalpoor stellar population indicates that the ejecta from numerous

9 http://WWW . inspect-stars . com/ 


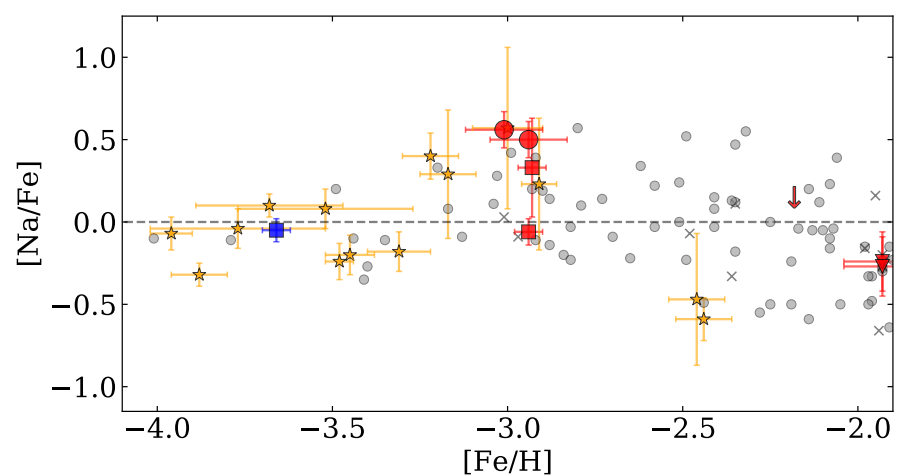

Fig. 4. Sodium-to-iron ratio as a function of $[\mathrm{Fe} / \mathrm{H}]$ are shown for metalpoor stars in Sextans, Sculptor, and MW halo stars. The symbols are the same as in Fig. 6. The stars studied in this paper are the large red symbols.

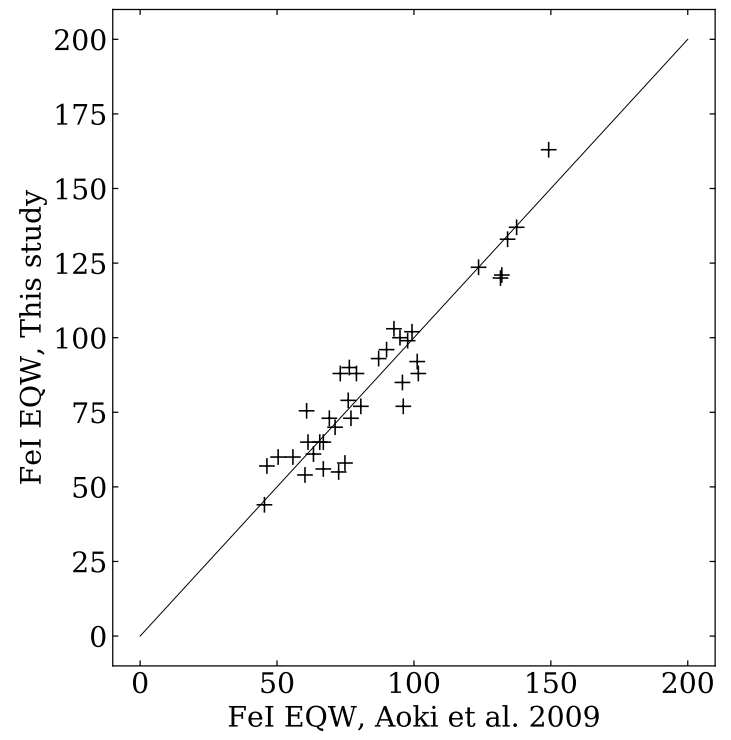

Fig. 5. Comparison for star S11-37 between Aoki et al. (2009) and our analysis on the measured Fe I EWs in common.

massive stars contributed to the metallicity of the interstellar medium (ISM), as indicated by the low scatter around the mean $[\mathrm{alpha} / \mathrm{Fe}]$ value at low metallicity. As pointed out by Audouze \& Silk (1995), the chemical composition of the ejecta from a supernova $(\mathrm{SN})$ depends on the mass of the progenitor, which means that the smaller the number of SNe that contributed to the ISM composition, the larger the abundance dispersion of the ISM. Even though this is further complicated by possible differences in mixing efficiency, we therefore expect that the abundance dispersion increases with decreasing stellar mass of a galaxy. Thus the abundance dispersion would be minimal in the MW, and higher but still relatively low in dSph galaxies. At low metallicity $([\mathrm{Fe} / \mathrm{H}] \lesssim-2.5)$, most members of $\mathrm{dSph}$ galaxies follow the same plateau as the MW halo stars (see, e.g., Jablonka et al. 2015 for Sculptor). Nevertheless, even in the relatively massive Sculptor dSph galaxy (with a stellar mass of $2.3 \times 10^{6} M_{\odot}$, McConnachie 2012), about one to three stars in this metallicity range have $[\alpha / \mathrm{Fe}] \leq 0.00$ (Fig. 6). The question still remains whether lower mass classical dSphs, such as Sextans and Carina, have a higher dispersion at fixed metallicity. In the case of Carina this is expected because of its star formation history, which is characterized by at least three distinct

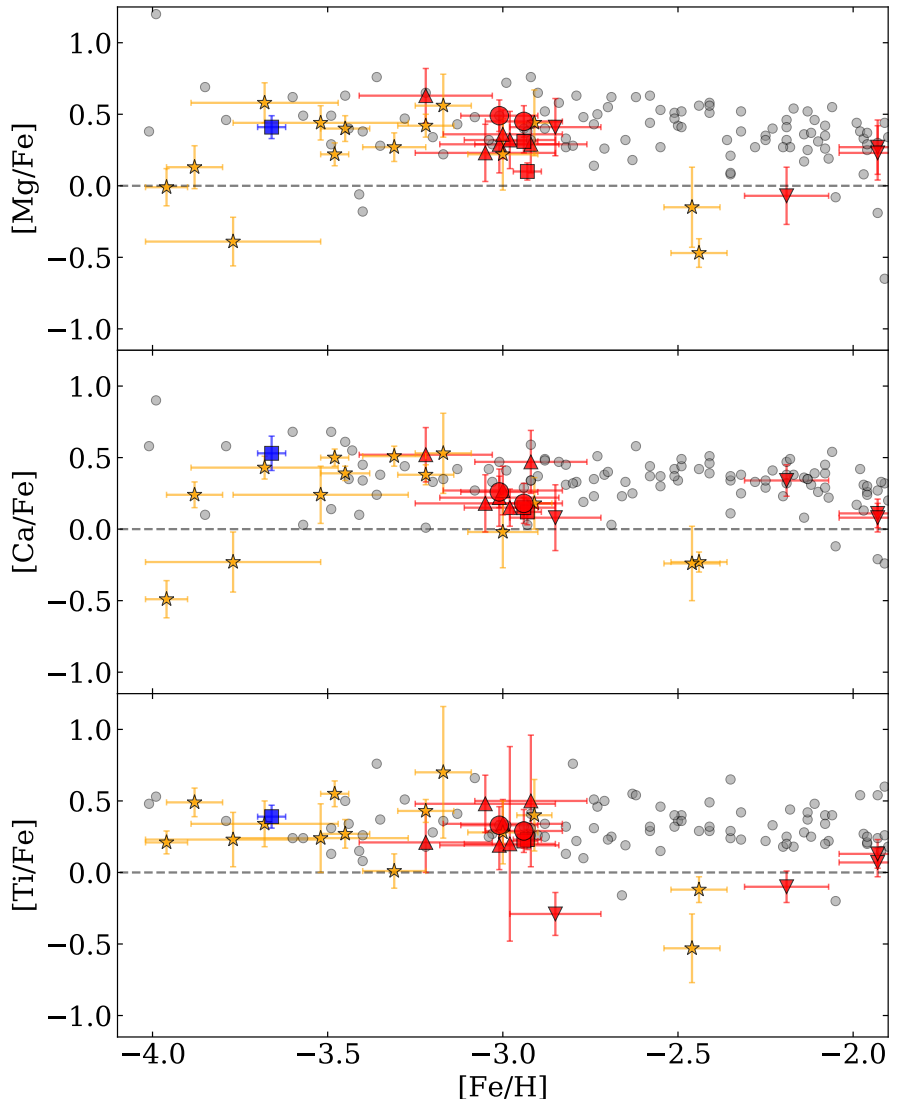

Fig. 6. Abundance ratios for the $\alpha$ elements $\mathrm{Mg}, \mathrm{Ca}$, and $\mathrm{Ti}$ (from top to bottom $)$ as a function of $[\mathrm{Fe} / \mathrm{H}]$. Sextans stars are large red symbols. The new EMP stars studied in this paper are the red circles. The sample of Aoki et al. (2009) that we reanalyzed is shown as red triangles. Data from Shetrone et al. (2001b) are upside-down triangles. Gray dots are literature data for MW halo stars (Venn et al. 2004; Cohen et al. 2013; Yong et al. 2013; Ishigaki et al. 2013). Orange and blue symbols refer to RGB stars observed in Sculptor (Jablonka et al. 2015; Tafelmeyer et al. 2010; Starkenburg et al. 2013; Simon et al. 2015) and Fornax (Tafelmeyer et al. 2010), respectively.

bursts (Hurley-Keller et al. 1998; Santana et al. 2016) that so far have been interpreted as resulting from interactions with the MW (Fabrizio et al. 2011, 2016; Pasetto et al. 2011). In Sextans, the observed dispersion in $[\alpha / \mathrm{Fe}]$, when data from Aoki et al. (2009) and Tafelmeyer et al. (2010) are considered, has been attributed to the effect that fewer SNe enriched the ISM from which the observed stars were born, and that pockets of ISM with various abundances coexist.

In the newly discovered EMPs observed with UVES, we measure an overabundance in $[\alpha / \mathrm{Fe}] \sim+0.4$ dex (see Fig. 6), which is comparable with the typical $[\alpha / \mathrm{Fe}]$ value observed in the halo of the MW. This is in stark contrast with the result of Aoki et al. (2009), who obtained solar $[\alpha / \mathrm{Fe}]$ ratios for the majority of their sample.

Because scatter can be artificially introduced when results from the different analyses are used, we applied the same method as we followed for the newly discovered EMPS to the literature sample. This allows for a fair and homogeneous comparison between the LTE abundances measured from Sextans stars and those observed in the Galactic halo.

In order to investigate into this apparent discrepancy, we therefore started by comparing our measured EWs with those presented in Aoki et al. (2009). For this exercise, we considered 
Table 5. Comparison of the derived $\log (\mathrm{Mg})$ abundances when all lines are retained, including the strong lines (SL), and when these strongest lines (noSL) are removed.

\begin{tabular}{lccc}
\hline \hline Star & $\log (\mathrm{Mg})_{\mathrm{SL}}$ & $\log (\mathrm{Mg})_{\mathrm{noSL}}$ & $\Delta(\log (\mathrm{Mg}))$ \\
\hline S10-14 & 4.63 & 4.88 & +0.25 \\
S11-13 & 4.53 & 4.78 & +0.25 \\
S11-37 & 4.71 & 4.94 & +0.23 \\
S12-28 & 4.95 & 4.96 & +0.01 \\
S14-98 & 4.86 & 4.96 & +0.10 \\
S15-19 & 5.22 & 5.01 & -0.21 \\
\hline
\end{tabular}

the star S11-37, which has the lowest metallicity in the group characterized by the low $\alpha$-element abundances. We retrieved the reduced spectra (eight exposures of 1800s for each, obtained in the blue and red arms of the Subaru High-Dispersion Spectrograph) from the JVO database ${ }^{10}$ and applied the same procedure as described in Sect. 2.2, with small adjustments to the HDS data. Briefly, the exposures were combined with IRAF, but the orders were extracted and fit individually with DAOSPEC in order to avoid any continuum modulation. Figure 5 shows that the EWs measured using our approach agree excellently well with those listed in Aoki et al. (2009). We therefore decided to use the Aoki et al. (2009) EWs to rederive the abundances as described in Sect. 3.

The star S15-19, with the lowest metallicity in the dataset of Aoki et al. (2009), has been re-observed and re-discussed by Honda et al. (2011) and has been confirmed to be a CEMP-s star. For the homogeneous reanalysis we used the new EWs measured by Honda et al. (2011).

The two analyses show some differences. First, Aoki et al. (2009) used the Kurucz (1993) atmosphere models while we use the MARCS 1D spherical models. Second, Aoki et al. (2009) determined the stellar effective temperatures by adopting the $V-K$ colour index (combined with a color-temperature calibration), while we derived our temperatures by minimizing the trend of Fe I abundances versus their excitation potential $\left(\chi_{\mathrm{exc}}\right)$.

This different approach is reflected in the mean difference in the atmospheric parameters $\Delta$ (this study - Aoki et al.) of $-65 \mathrm{~K},-0.2 \mathrm{cgs},-0.7 \mathrm{~km} . \mathrm{s}^{-1}$ and $-0.2 \mathrm{dex}$, in $T_{\text {eff }}, \log (g), v_{\mathrm{t}}$, and $[\mathrm{Fe} / \mathrm{H}]$, respectively.

Abundances of the $\alpha$-elements echo this change in metallicity determination, but the largest difference between the two studies lies in the selection of lines that were used in the analysis. Specifically, Mg abundances in Aoki et al. (2009) are typically derived from three to four lines, including two very strong lines (at 5172 and $5183 \AA$ ) with EWs that exceed $>150 \mathrm{~m} \AA$. Strong lines are not reliable when a Gaussian fitting routine is employed, and they give systematically lower $\mathrm{Mg}$ abundances than the $\mathrm{Mg}$ line at $5528 \AA$ (with typical EW $\sim 55 \mathrm{~m} \AA$ ). They were therefore excluded from the analysis. As to whether $[\mathrm{Fe} / \mathrm{H}]$ or $[\mathrm{Mg} / \mathrm{H}]$ drives the change in $[\mathrm{Mg} / \mathrm{Fe}]$, we stress that retaining the strong $\mathrm{Mg}$ lines in a pure EW analysis (hence without proper synthesis) does affect the final result. This is clearly seen when we compare the $\log (\mathrm{Mg})$ (absolute) abundances (1) when all lines are retained and (2) when the very strong lines are removed (see Table 5). Had we retained the very strong lines, the $[\mathrm{Mg} / \mathrm{Fe}]$ ratios would only have changed by 0.05 to 0.14 compared to Aoki et al. (2009).

\footnotetext{
$\overline{10}$ https://jvo.nao.ac.jp/portal/subaru/hds.do
}

Figure 6 shows the measured abundances of $\alpha$-elements from our newly observed EMPs and the reanalysis of Aoki et al. (2009) stars. The two Sextans stars presented in the previous paper of this series (Tafelmeyer et al. 2010) are also shown.

Sextans stars have $[\mathrm{Mg} / \mathrm{Fe}]$ abundance ratios that nicely follow the trend of the Galactic halo. We do not confirm the presence of a low- $\alpha$ population as claimed in Aoki et al. (2009). The only exception is star S36 from Shetrone et al. (2001b) with $[\mathrm{Mg} / \mathrm{Fe}]=-0.07 \pm 0.20$, based on two strong lines fit by Gaussians on a spectrum with $S / N=13$ only. Stars with homogeneously derived abundances (e.g., large red symbols, triangles, and squares in Fig. 6) also appear to be enhanced in Ti II at the level observed in $\mathrm{Mg}$ and $\mathrm{Ca}$ with a normal $\sim 0.2$ dex dispersion.

\subsection{Iron-peak elements}

Figure 7 presents the abundance ratios of scandium, nickel, cobalt, zinc, chromium, and manganese as a function of metallicity. These elements are all produced by explosive nucleosynthesis.

The scandium abundances of our stars follow the MW halo trend very closely. The Sc production is dominated by SNeII (e.g., Woosley et al. 2002; Battistini \& Bensby 2015), therefore the trend of Sc II/Fe with iron nicely follows the run of the $\alpha$ elements with metallicity.

$\mathrm{Ni}$ and $\mathrm{Co}$ can also be produced by SNeIa (e.g., Travaglio et al. 2005; Kirby et al. 2018). However, the contribution by SNeIa starts to dominate the chemical evolution of the galaxy at higher metallicities $([\mathrm{Fe} / \mathrm{H}] \geq-2$; Theler et al. 2020). The behavior of $\mathrm{Ni} / \mathrm{Fe}$ in the low-metallicity range investigated here can therefore be attributed to $\mathrm{Ni}$ production by complete and incomplete Si burning.

$\mathrm{Co}$ and $\mathrm{Zn}$ are produced by the complete $\mathrm{Si}$ burning when the peak temperature of the shock material is above $5 \times 10^{9} \mathrm{~K}$ (Nomoto et al. 2013). The [Co I/Fe] ratios observed in our Sextans stars cover the lower tail of the distribution in the MW halo, similarly to the Fornax and three of the Sculptor EMPS. This might simply be an observational bias in our data sample because in dSphs we normally observe bright evolved RGB stars, which have lower temperatures and surface gravities than those in the MW halo. Additionally, these abundances should be corrected for the NLTE effect. These corrections depend on the stellar parameters as well (Bergemann et al. 2010; Kirby et al. 2018). It is interesting to note that the lowest $[\mathrm{Co} / \mathrm{Fe}]$ EMPS in Sculptor are also the coolest, in the same temperature range $\sim 4500 \mathrm{~K}$ as in Sextans (Starkenburg et al. 2013; Jablonka et al. 2015). The Fornax EMPS is even cooler ( $4300 \mathrm{~K}$, Tafelmeyer et al. 2010) Unfortunately, no NLTE corrections for the range of atmospheric parameters of our stars are available, which would help shed light on the relative strength of the corrections.

The $\mathrm{Zn}$ abundances are measured from a weak line (with $\mathrm{EW}$ of 23 to $30 \mathrm{~m} \AA$ ) at $4810 \AA$. However, because the ( 50) S/N of the spectra around the $\mathrm{Zn}$ feature is relatively high, we were able to measure accurate $\mathrm{Zn}$ abundances. This is the first unambiguous measurement of $\mathrm{Zn}$ at low metallicity in a classical dwarf. Simon et al. (2015) reported on the detection of $\mathrm{Zn}$ in the EMP Scl07-49 in Sculptor. However, for the same star and the same spectrum, Tafelmeyer et al. (2010) have concluded only an upper limit. The measured $\mathrm{Zn}$ abundances perfectly follow the $[\mathrm{Zn} / \mathrm{Fe}]$ versus $[\mathrm{Fe} / \mathrm{H}]$ observed in the $\mathrm{MW}$ very metal-poor stars, with an enhancement up to $\sim 0.7 \mathrm{dex}$. The production sites of $\mathrm{Zn}$ remain uncertain. The increasing enhancement at decreasing metallicity suggests that $\mathrm{Zn}$ was produced efficiently at the very early stages of the galaxy formation, likely in SNeII. The production 

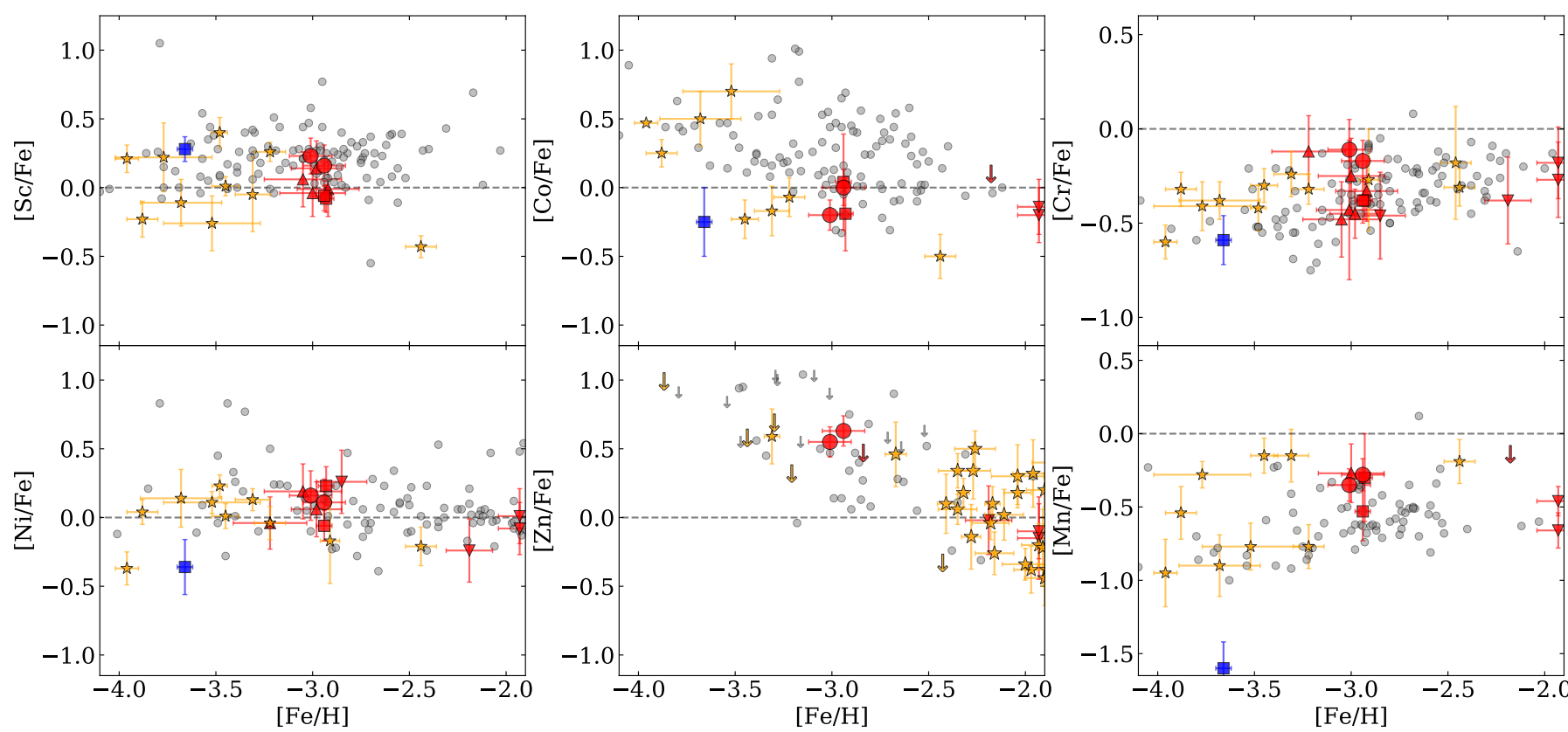

Fig. 7. From left to right, top to bottom: $[\mathrm{Sc} / \mathrm{Fe}],[\mathrm{Co} / \mathrm{Fe}],[\mathrm{Cr} / \mathrm{Fe}],[\mathrm{Ni} / \mathrm{Fe}],[\mathrm{Zn} / \mathrm{Fe}]$, and $[\mathrm{Mn} / \mathrm{Fe}]$ for metal-poor stars in Sextans, Sculptor, $\mathrm{Fornax}$, and MW halo stars. The symbols are the same as in Fig. 6. The stars studied in this paper are the large red symbols.

through classical SNeII was shown to be insufficient to explain the observed [Zn/Fe] (Hirai et al. 2018; Tsujimoto \& Nishimura 2018), however.

In the incomplete Si-burning region, the after-decay products include chromium and manganese (Nomoto et al. 2013). Figure 7 shows that the $[\mathrm{Cr} / \mathrm{Fe}]$ and $[\mathrm{Mn} / \mathrm{Fe}]$ trends with $[\mathrm{Fe} / \mathrm{H}]$ in Sextans stars follow the Galactic trend well.

Bergemann \& Cescutti (2010) have shown that in stars over the wide range of metallicities between $-3.2 \leq[\mathrm{Fe} / \mathrm{H}] \leq-0.5$, the $[\mathrm{Cr} / \mathrm{Fe}]$ ratio computed in NLTE is roughly solar, which is consistent with current views of the production of these iron peak elements in supernovae. This means that the apparent increase in $[\mathrm{Cr} / \mathrm{Fe}]$ ratios with metallicity in MW stars in Fig. 7 is not real but rather due to the LTE approximation. NLTE corrections are not available for the range of stellar APs explored here. Nonetheless, NLTE corrections on $\mathrm{Cr}$ abundances are expected to be positive for bright giants (Mashonkina, priv. comm.).

\subsection{Neutron-capture elements}

The heavy elements (heavier than $\mathrm{Zn}$ ) are synthesized through two main processes. The $s$-process operates by slow neutron capture on seed nuclei on a long timescale (i.e., the neutron capture is slower than the $\beta$ decay of the affected nucleus). The stellar sources for $s$-process production are asymptotic giant branch (AGB) stars (e.g., Busso et al. 1999; Käppeler et al. 2011; Bisterzo et al. 2012). The $r$-process instead occurs on a very short timescale in violent events (e.g., Cameron 1957). High-entropy neutrino-driven winds of core-collapse supernovae (CCSNe) have traditionally been considered the sites of $r$-process nucleosynthesis (e.g., Sneden et al. 2008). However, they have been ruled out as responsible for the origin of the main $r$-process elements by observations and simulations (Wanajo 2013; Macias \& Ramirez-Ruiz 2018), and other exotic types of CCSNe have been put forward (e.g., magnetorotational SNe; Nishimura et al. 2015). The recent LIGO/Virgo discovery of gravitational waves from the neutron star merger (NSM) GW170817 (Abbott et al. 2017) and the follow-up

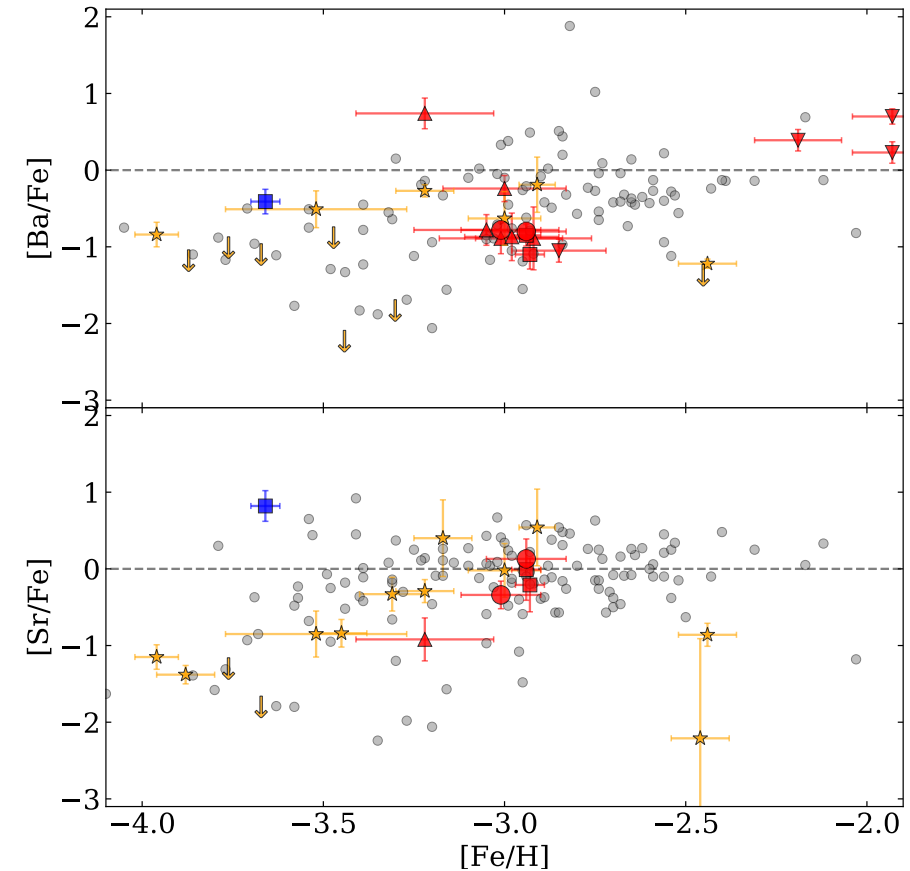

Fig. 8. Neutron-capture elements: Barium-to-iron ratio at the top and strontium-to-iron ratio at the bottom, as a function of $[\mathrm{Fe} / \mathrm{H}]$ in Sextans shown in red, compared to the MW halo stars in gray. The large circles represent the new sample in Sextans. Orange symbols refer to Sculptor.

kilonova observations (e.g., Pian et al. 2017) have shown that NSMs produce a copious amount of $r$-process material (e.g., Lattimer \& Schramm 1974; Freiburghaus et al. 1999; Côté et al. 2017). This notion is also supported by the detection of $r$ process enrichment in the ultra-faint dwarf (UFD) Reticulum II (Ji et al. 2016; Roederer et al. 2016). However, the evidence that $r$-process is found also in low-mass systems where NSMs should be rare suggests that there might be different sites or conditions for the production of $r$-process elements (Travaglio et al. 2004; 


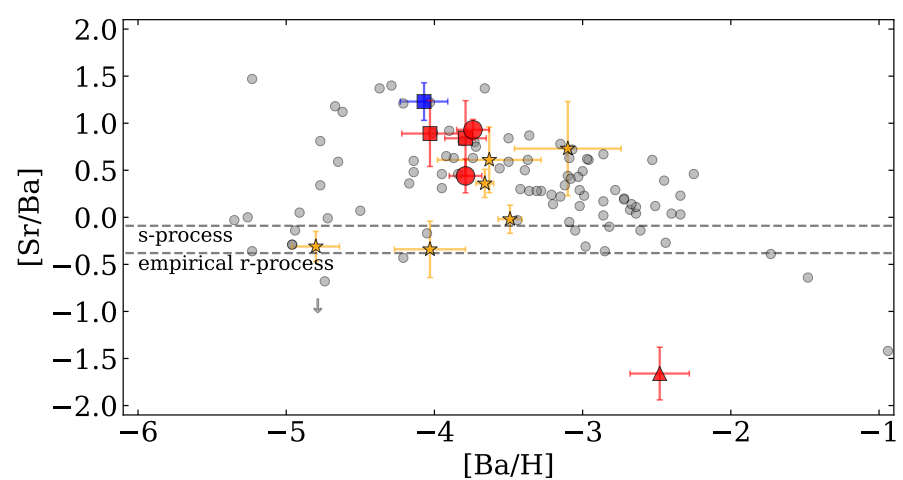

Fig. 9. Barium-to-strontium ratio as a function of $[\mathrm{Ba} / \mathrm{H}]$ in Sextans shown in red, compared to the MW halo stars presented in gray. Sculptor is shown in orange, and Fornax is in blue. References are the same as in Fig. 6. The $s$-process and empirical $r$-process limits are shown with dashed lines (Mashonkina et al. 2017b).

Jablonka et al. 2015; Mashonkina et al. 2017b; Hansen et al. 2018).

These two distinct processes produce generally different isotopes of a given heavy element, and different element ratios. Two neutron-capture elements can be measured in our stars: barium and strontium. At very low metallicity (i.e., $[\mathrm{Fe} / \mathrm{H}] \leq-2.5$ ), a significant enrichment by AGBs is not expected. In our EMP stars, we therefore expect a pure $r$-process origin for the neutroncapture elements.

Europium can be formed basically only through the $r$-process. However, Eu measurements in EMP stars are rare because Eu lines are very weak at low-metallicities. We were not able to detect clean Eu features in our spectra. Nonetheless, $[\mathrm{Eu} / \mathrm{Fe}]$ seems to correlate well with $[\mathrm{Ba} / \mathrm{Fe}]$ for $[\mathrm{Fe} / \mathrm{H}]$ for metallicities $[\mathrm{Fe} / \mathrm{H}] \leq-2.5$ (e.g., Mashonkina et al. 2010; Spite \& Spite 2014). At very low metallicity, even Ba has therefore been formed by the $r$-process.

$\mathrm{Sr}$ and $\mathrm{Ba}$ abundances are shown in Fig. 8 as a function of metallicity. As found earlier, $[\mathrm{Ba} / \mathrm{Fe}]$ is generally below solar in the EMP stars, with a significant scatter (Travaglio et al. 2004; François et al. 2007). In the same plot, we also show abundances for stars observed at high resolution in the MW halo, Fornax, and Sculptor (see Fig. 6 for full references). In the MW halo sample a high dispersion in both $[\mathrm{Sr} / \mathrm{Fe}]$ and $[\mathrm{Ba} / \mathrm{Fe}]$ can be observed at metallicities lower than $[\mathrm{Fe} / \mathrm{H}] \leq-2.8$ and -2.5 for $\mathrm{Sr}$ and $\mathrm{Ba}$; respectively (e.g., Andrievsky et al. 2009, 2010; Hansen et al. 2013; Mashonkina et al. 2017b). Above this metallicity, [Sr/Fe] and $[\mathrm{Ba} / \mathrm{Fe}]$ steadily become solar, and the dispersion is largely diminished.

Figure 8 shows that except for S15-19, which is a carbonrich star with evidence for s-process enrichment (Honda et al. 2011), all Sextans EMPS so far investigated at very high resolution have subsolar $[\mathrm{Ba} / \mathrm{Fe}]$ ratios at $[\mathrm{Fe} / \mathrm{H}] \sim-3$, to a level that is close to the level encountered at much lower metallicities for Fornax and Sculptor and in the UFDs (Simon 2019), hence tracing the initial trend between $\mathrm{Fe}$ and $\mathrm{Ba}$, most likely arising from CCSNe. This concentration is most likely a coincidence because at higher metallicities, $[\mathrm{Ba} / \mathrm{Fe}]$ reaches the solar plateau. It is useful to appreciate the difference in $\mathrm{Sr}$ and $\mathrm{Ba}$ behaviors in general. For the same stars, $[\mathrm{Sr} / \mathrm{Fe}]$ is clumped around the solar value in a similar way as the MW halo population, suggesting similar enrichment processes for strontium.

Figure 9 shows the run of the $[\mathrm{Sr} / \mathrm{Ba}]$ ratio plotted against $[\mathrm{Ba} / \mathrm{H}]$. If $\mathrm{Ba}$ and $\mathrm{Sr}$ were formed by the same process, their ratio should not vary with $[\mathrm{Ba} / \mathrm{H}]$. All Sextans stars that so far have been observed at high resolution, except for the $s$-process star S15-19 (Honda et al. 2011), are perfectly located at the top of the decreasing branch of $[\mathrm{Sr} / \mathrm{Ba}]$ with $[\mathrm{Ba} / \mathrm{H}]$. This confirms that the source responsible for the production of lighter $(\mathrm{Sr})$ neutron-capture elements is at work at earlier times than the processes that produce heavier ( $\mathrm{Ba}$ ) neutron-capture elements (e.g., François et al. 2007; Mashonkina et al. 2017b; Spite et al. 2018; Frebel 2018; Hansen et al. 2018).

\section{Summary}

We have presented the analysis of high-resolution spectra of two metal-poor stars in the dwarf spheroidal galaxy Sextans, including the abundance derivation of 18 chemical elements. In particular, we provide the first unambiguous measurement of $\mathrm{Zn}$ in a classical $\mathrm{dSph}$ in this metallicity range. These stars are confirmed as some of the most metal-poor stars known in Sextans. Literature spectra originally presented in Aoki et al. (2009) were reinvestigated in a homogeneous manner, and abundances for Fe I, Fe II, Mg, Ca, Sc II, Ti I, Ti II, Cr, and Ba II were rederived. This full sample significantly increases the number of stars in the low-metallicity range and gives new clues on the formation of Sextans. In particular, we demonstrated that the Sextans metalpoor population follows the MW halo-like plateau at $[\alpha / \mathrm{Fe}] \sim$ 0.4 with a normal scatter. This is different from previous results.

Most of the iron-peak elements are aligned with the MW halo distribution. Only cobalt is slightly depleted. We suggest on observational grounds that $[\mathrm{Co} / \mathrm{Fe}]$ might scale with the stellar effective temperature and that differential NLTE corrections would place the MW and dSph populations on the same scale.

The four Sextans (non-carbon rich) EMPS analyzed at high resolution have $[\mathrm{Fe} / \mathrm{H}] \sim-3$ and $[\mathrm{Ba} / \mathrm{Fe}] \sim-1$. This corresponds to the $\mathrm{Ba}$ floor seen at $[\mathrm{Fe} / \mathrm{H}]$ below -3.5 in the $\mathrm{MW}$ halo, in the UFDs, and in Sculptor. At this metallicity and this Ba enrichment, $[\mathrm{Sr} / \mathrm{Fe}]$ is already solar. This confirms that the source responsible for the production of the light neutron-capture elements precedes the production of the heavier ones. It also shows that this source is already efficient at the galaxy mass of Sextans.

Acknowledgements. The authors warmly thank Lyudmila Mashonkina for useful discussions on the NLTE corrections. The authors acknowledge the support and funding of the International Space Science Institute (ISSI) through the International Team "Pristine". CL acknowledges financial support from the Swiss National Science Foundation (Ambizione grant PZ00P2_168065). GB acknowledges financial support through the grant (AEI/FEDER, UE) AYA2017-89076-P, as well as by the Ministerio de Ciencia, Innovacion y Universidades (MCIU), through the State Budget and by the Consejeria de Economia, Industria, Comercio y Conocimiento of the Canary Islands Autonomous Community, through the Regional Budget.

\section{References}

Abbott, B. P., Abbott, R., Abbott, T. D., et al. 2017, Phys. Rev. Lett., 119, 161101 Alonso, A., Arribas, S., \& Martínez-Roger, C. 1999, A\&AS, 140, 261 Alvarez, R., \& Plez, B. 1998, A\&A, 330, 1109

Amarsi, A. M., Nissen, P. E., Asplund, M., Lind, K., \& Barklem, P. S. 2019, A\&A, 622, L4

Andrievsky, S. M., Spite, M., Korotin, S. A., et al. 2009, A\&A, 494, 1083 Andrievsky, S. M., Spite, M., Korotin, S. A., et al. 2010, A\&A, 509, A88

Aoki, W., Beers, T. C., Christlieb, N., et al. 2007, ApJ, 655, 492

Aoki, W., Arimoto, N., Sadakane, K., et al. 2009, A\&A, 502, 569

Asplund, M., Grevesse, N., Sauval, A. J., \& Scott, P. 2009, ARA\&A, 47, 481

Audouze, J., \& Silk, J. 1995, ApJ, 451, L49

Battaglia, G., Tolstoy, E., Helmi, A., et al. 2011, VizieR Online Data Catalog: VII/41

Battistini, C., \& Bensby, T. 2015, A\&A, 577, A9 
Beers, T. C., \& Christlieb, N. 2005, ARA\&A, 43, 531

Bergemann, M., \& Cescutti, G. 2010, A\&A, 522, A9

Bergemann, M., Pickering, J. C., \& Gehren, T. 2010, MNRAS, 401, 1334

Bettinelli, M., Hidalgo, S. L., Cassisi, S., Aparicio, A., \& Piotto, G. 2018, MNRAS, 476, 71

Bisterzo, S., Gallino, R., Straniero, O., Cristallo, S., \& Käppeler, F. 2012, MNRAS, 422, 849

Breddels, M. A., \& Helmi, A. 2013, A\&A, 558, A35

Busso, M., Gallino, R., \& Wasserburg, G. J. 1999, ARA\&A, 37, 239

Cameron, A. G. W. 1957, AJ, 62, 9

Cardelli, J. A., Clayton, G. C., \& Mathis, J. S. 1989, ApJ, 345, 245

Chiti, A., Simon, J. D., Frebel, A., et al. 2018, ApJ, 856, 142

Cicuéndez, L., \& Battaglia, G. 2018, MNRAS, 480, 251

Cicuéndez, L., Battaglia, G., Irwin, M., et al. 2018, A\&A, 609, A53

Cohen, J. G., \& Huang, W. 2009, ApJ, 701, 1053

Cohen, J. G., Christlieb, N., Thompson, I., et al. 2013, ApJ, 778, 56

Côté, B., Belczynski, K., Fryer, C. L., et al. 2017, ApJ, 836, 230

Dekker, H., D’Odorico, S., Kaufer, A., Delabre, B., \& Kotzlowski, H. 2000 in Society of Photo-Optical Instrumentation Engineers (SPIE) Conference Series, eds. M. Iye, \& A. F. Moorwood, Proc. SPIE, 4008, 534

Ezzeddine, R., Frebel, A., \& Plez, B. 2017, ApJ, 847, 142

Fabrizio, M., Nonino, M., Bono, G., et al. 2011, PASP, 123, 384

Fabrizio, M., Bono, G., Nonino, M., et al. 2016, ApJ, 830, 126

François, P., Depagne, E., Hill, V., et al. 2007, A\&A, 476, 935

Frebel, A. 2018, Ann. Rev. Nucl. Part. Sci., 68, 237

Frebel, A., \& Norris, J. E. 2015, ARA\&A, 53, 631

Freiburghaus, C., Rosswog, S., \& Thielemann, F. K. 1999, ApJ, 525, L121

Gustafsson, B., Edvardsson, B., Eriksson, K., et al. 2008, A\&A, 486, 951

Hansen, C. J., Bergemann, M., Cescutti, G., et al. 2013, A\&A, 551, A57

Hansen, T. T., Holmbeck, E. M., Beers, T. C., et al. 2018, ApJ, 858, 92

Hill, V., Skúladóttir, Á., Tolstoy, E., et al. 2019, A\&A, 626, A15

Hirai, Y., Saitoh, T. R., Ishimaru, Y., \& Wanajo, S. 2018, ApJ, 855, 63

Honda, S., Aoki, W., Arimoto, N., \& Sadakane, K. 2011, PASJ, 63, 523

Hurley-Keller, D., Mateo, M., \& Nemec, J. 1998, AJ, 115, 1840

Irwin, M. J., Bunclark, P. S., Bridgeland, M. T., \& McMahon, R. G. 1990, MNRAS, 244, 16P

Ishigaki, M. N., Aoki, W., \& Chiba, M. 2013, ApJ, 771, 67

Jablonka, P., North, P., Mashonkina, L., et al. 2015, A\&A, 583, A67

Ji, A. P., Frebel, A., Chiti, A., \& Simon, J. D. 2016, Nature, 531, 610

Käppeler, F., Gallino, R., Bisterzo, S., \& Aoki, W. 2011, Rev. Mod. Phys., 83, 157

Karachentsev, I. D., Karachentseva, V. E., Huchtmeier, W. K., \& Makarov, D. I. 2004, AJ, 127, 2031

Kirby, E. N., \& Cohen, J. G. 2012, AJ, 144, 168

Kirby, E. N., Guo, M., Zhang, A. J., et al. 2015, ApJ, 801, 125

Kirby, E. N., Xie, J. L., Guo, R., Kovalev, M., \& Bergemann, M. 2018, ApJS, 237, 18

Kupka, F., Piskunov, N., Ryabchikova, T. A., Stempels, H. C., \& Weiss, W. W. 1999, A\&AS, 138, 119

Kupka, F. G., Ryabchikova, T. A., Piskunov, N. E., Stempels, H. C., \& Weiss, W. W. 2000, Baltic Astron., 9, 590

Kurucz, R. L. 1993, in IAU Colloq. 138: Peculiar versus Normal Phenomena in A-type and Related Stars, eds. M. M. Dworetsky, F. Castelli, \& R. Faraggiana, ASP Conf. Ser., 44, 87

Lattimer, J. M., \& Schramm, D. N. 1974, ApJ, 192, L145

Lee, M. G., Park, H. S., Park, J.-H., et al. 2003, AJ, 126, 2840

Lee, M. G., Yuk, I.-S., Park, H. S., Harris, J., \& Zaritsky, D. 2009, ApJ, 703, 692

Letarte, B., Hill, V., Tolstoy, E., et al. 2010, A\&A, 523, A17

Lind, K., Asplund, M., Barklem, P. S., \& Belyaev, A. K. 2011, A\&A, 528, A103
Łokas, E. L. 2009, MNRAS, 394, L102

Macias, P., \& Ramirez-Ruiz, E. 2018, ApJ, 860, 89

Magain, P. 1984, A\&A, 134, 189

Mashonkina, L., Christlieb, N., Barklem, P. S., et al. 2010, A\&A, 516, A46

Mashonkina, L., Jablonka, P., Pakhomov, Y., Sitnova, T., \& North, P. 2017a, A\&A, 604, A129

Mashonkina, L., Jablonka, P., Sitnova, T., Pakhomov, Y., \& North, P. 2017b, A\&A, 608, A89

Mateo, M., Fischer, P., \& Krzeminski, W. 1995, AJ, 110, 2166

McConnachie, A. W. 2012, AJ, 144, 4

Muñoz, R. R., Côté, P., Santana, F. A., et al. 2018, ApJ, 860, 66

Nishimura, N., Takiwaki, T., \& Thielemann, F.-K. 2015, ApJ, 810, 109

Noguchi, K., Aoki, W., Kawanomoto, S., et al. 2002, PASJ, 54, 855

Nomoto, K., Kobayashi, C., \& Tominaga, N. 2013, ARA\&A, 51, 457

North, P., Cescutti, G., Jablonka, P., et al. 2012, A\&A, 541, A45

Okamoto, S., Arimoto, N., Tolstoy, E., et al. 2017, MNRAS, 467, 208

Pasetto, S., Grebel, E. K., Berczik, P., Chiosi, C., \& Spurzem, R. 2011, A\&A, 525, A99

Pian, E., D'Avanzo, P., Benetti, S., et al. 2017, Nature, 551, 67

Piskunov, N. E., Kupka, F., Ryabchikova, T. A., Weiss, W. W., \& Jeffery, C. S. 1995, A\&AS, 112, 525

Placco, V. M., Frebel, A., Beers, T. C., \& Stancliffe, R. J. 2014, ApJ, 797, 21

Plez, B. 2012, Astrophysics Source Code Library [record ascl:1205.004]

Press, W. H., \& Schechter, P. 1974, ApJ, 187, 425

Prochaska, J. X., \& McWilliam, A. 2000, ApJ, 537, L57

Ramírez, I., \& Meléndez, J. 2005, ApJ, 626, 465

Roederer, I. U., Mateo, M., Bailey, J. I., et al. 2016, AJ, 151, 82

Ryabchikova, T. A., Piskunov, N. E., Kupka, F., \& Weiss, W. W. 1997, Baltic Astron., 6, 244

Salvadori, S., Skúladóttir, Á., \& Tolstoy, E. 2015, MNRAS, 454, 1320

Santana, F. A., Muñoz, R. R., de Boer, T. J. L., et al. 2016, ApJ, 829, 86

Shetrone, M. D., Côté, P., \& Sargent, W. L. W. 2001a, ApJ, 548, 592

Shetrone, M. D., Côté, P., \& Sargent, W. L. W. 2001b, ApJ, 548, 592

Shetrone, M. D., Smith, G. H., Stanford, L. M., Siegel, M. H., \& Bond, H. E. 2013, AJ, 145, 123

Simon, J. D. 2019, ARA\&A, 57, 375

Simon, J. D., Jacobson, H. R., Frebel, A., et al. 2015, ApJ, 802, 93

Skúladóttir, Á., Tolstoy, E., Salvadori, S., et al. 2015, A\&A, 574, A129

Sneden, C., Cowan, J. J., \& Gallino, R. 2008, ARA\&A, 46, 241

Spite, M., \& Spite, F. 2014, Astron. Nachr., 335, 65

Spite, F., Spite, M., Barbuy, B., et al. 2018, A\&A, 611, A30

Springel, V., Frenk, C. S., \& White, S. D. M. 2006, Nature, 440, 1137

Starkenburg, E., Hill, V., Tolstoy, E., et al. 2010, A\&A, 513, A34

Starkenburg, E., Hill, V., Tolstoy, E., et al. 2013, A\&A, 549, A88

Stetson, P. B., \& Pancino, E. 2008, PASP, 120, 1332

Tafelmeyer, M., Jablonka, P., Hill, V., et al. 2010, A\&A, 524, A58

Theler, R., Jablonka, P., Lardo, C., et al. 2020, A\&A, 642, A176

Tolstoy, E., Hill, V., \& Tosi, M. 2009, ARA\&A, 47, 371

Travaglio, C., Gallino, R., Arnone, E., et al. 2004, ApJ, 601, 864

Travaglio, C., Hillebrandt, W., \& Reinecke, M. 2005, A\&A, 443, 1007

Tsujimoto, T., \& Nishimura, N. 2018, ApJ, 863, L27

Van der Swaelmen, M., Hill, V., Primas, F., \& Cole, A. A. 2013, A\&A, 560, A44

Venn, K. A., Irwin, M., Shetrone, M. D., et al. 2004, AJ, 128, 1177

Walker, M. G., McGaugh, S. S., Mateo, M., Olszewski, E. W., \& Kuzio de Naray, R. 2010, ApJ, 717, L87

Wanajo, S. 2013, ApJ, 770, L22

White, S. D. M., \& Rees, M. J. 1978, MNRAS, 183, 341

Woosley, S. E., Heger, A., \& Weaver, T. A. 2002, Rev. Mod. Phys., 74, 1015

Yong, D., Norris, J. E., Bessell, M. S., et al. 2013, ApJ, 762, 26 
R. Lucchesi et al.: Homogeneity in the early chemical evolution of the Sextans dwarf spheroidal galaxy

\section{Appendix A: Additional table}

Table A.1. Line parameters, observed EWs, and elemental abundances. EWs in brackets are given as indication only; the quoted abundances are derived through spectral synthesis for these lines.

\begin{tabular}{|c|c|c|c|c|c|c|c|}
\hline El. & $\begin{array}{c}\lambda \\
{[\AA]}\end{array}$ & $\begin{array}{l}\chi_{\mathrm{ex}} \\
{[\mathrm{eV}]}\end{array}$ & $\log (g f)$ & \multicolumn{2}{|c|}{$\mathrm{EW}[\mathrm{mA}] \quad \log \epsilon(\mathrm{X})$} & $\begin{array}{r}\text { EW }[\mathrm{mA}] \quad \text { los } \\
\text { S11-97 }\end{array}$ & $(\mathrm{X})$ \\
\hline $\mathrm{C}(\mathrm{CH})$ & 4323 & & & & 4.96 & & 4.87 \\
\hline $\mathrm{NaI}$ & 5889.951 & 0.00 & 0.108 & $(198.9) \pm(16.3)$ & 3.80 & $(189.1) \pm(11.9)$ & 3.79 \\
\hline $\mathrm{NaI}$ & 5895.924 & 0.00 & -0.194 & $(162.3) \pm(9.9)$ & 3.80 & $(179.2) \pm(11.9)$ & 3.79 \\
\hline$M g$ & 3829.355 & 2.71 & -0.227 & - \pm- & - & $(176.0) \pm(14.6)$ & 5.09 \\
\hline $\operatorname{Mg} I$ & 3832.304 & 2.71 & 0.125 & $(190.7) \pm(17.8)$ & 5.13 & - \pm- & - \\
\hline$M g_{I}$ & 3838.294 & 2.72 & -0.351 & $(214.5) \pm(16.1)$ & 5.13 & $(221.2) \pm(18.0)$ & 5.10 \\
\hline$M g_{I}$ & 5172.684 & 2.71 & -0.450 & $(131.3) \pm(16.9)$ & 5.10 & $(181.6) \pm(14.1)$ & 5.04 \\
\hline $\mathrm{Mg}_{\mathrm{I}}$ & 5183.604 & 2.72 & -0.239 & $(146.8) \pm(17.2)$ & 5.11 & $(153.9) \pm(15.2)$ & 5.05 \\
\hline $\mathrm{Mg}_{\mathrm{I}}$ & 5528.405 & 4.35 & -0.498 & $(59.8) \pm(4.3)$ & 5.08 & $(62.9) \pm(4.7)$ & 5.12 \\
\hline $\mathrm{Al} \mathrm{I}$ & 3944.006 & 0.00 & -0.623 & $(110.1) \pm(20.4)$ & 2.95 & - \pm- & - \\
\hline $\mathrm{Al} \mathrm{I}$ & 3961.520 & 0.01 & -0.323 & $(137.6) \pm(11.7)$ & 2.98 & $(138.4) \pm(10.5)$ & 3.05 \\
\hline Si I & 3905.523 & 1.91 & -1.041 & (185.6) & - & (195.4) & - \\
\hline Si I & 4102.936 & 1.909 & -3.140 & $(89.6)$ & - & $(58.7)$ & - \\
\hline $\mathrm{CaI}$ & 4283.011 & 1.89 & -0.136 & $45.8 \pm 4.5$ & 3.42 & $49.1 \pm 4.4$ & 3.44 \\
\hline Ca I & 4318.651 & 1.90 & -0.139 & $41.7 \pm 5.0$ & 3.36 & - \pm- & - \\
\hline $\mathrm{Ca} \mathrm{I}$ & 4434.957 & 1.89 & -0.007 & - \pm- & - & $61.2 \pm 6.6$ & 3.51 \\
\hline $\mathrm{Ca} \mathrm{I}$ & 4454.779 & 1.90 & 0.258 & $79.4 \pm 7.6$ & 3.70 & $78.9 \pm 6.4$ & 3.61 \\
\hline $\mathrm{Ca}$ & 5265.556 & 2.52 & -0.113 & - \pm- & - & $24.9 \pm 2.7$ & 3.64 \\
\hline $\mathrm{Ca} \mathrm{I}$ & 5349.465 & 2.71 & -0.310 & - \pm- & - & $12.5 \pm 1.6$ & 3.69 \\
\hline Ca I & 5581.965 & 2.52 & -0.555 & $11.1 \pm 1.4$ & 3.67 & - \pm- & - \\
\hline $\mathrm{Ca}$ & 5588.749 & 2.53 & 0.358 & $37.1 \pm 3.2$ & 3.44 & $40.5 \pm 3.2$ & 3.46 \\
\hline $\mathrm{Ca} \mathrm{I}$ & 5857.451 & 2.93 & 0.240 & - \pm- & - & $19.9 \pm 2.1$ & 3.63 \\
\hline Ca I & 6102.723 & 1.88 & -0.793 & $34.5 \pm 3.1$ & 3.71 & $33.2 \pm 3.0$ & 3.64 \\
\hline Ca I & 6122.217 & 1.89 & -0.316 & $57.3 \pm 4.7$ & 3.63 & $59.7 \pm 4.5$ & 3.61 \\
\hline $\mathrm{CaI}$ & 6162.173 & 1.90 & -0.090 & $76.7 \pm 5.8$ & 3.75 & $75.5 \pm 5.7$ & 3.65 \\
\hline Ca I & 6439.075 & 2.53 & 0.390 & $47.8 \pm 5.0$ & 3.55 & $53.6 \pm 4.2$ & 3.60 \\
\hline Sc II & 4246.822 & 0.31 & 0.242 & $(128.0) \pm(11.5)$ & 0.33 & $(129.0) \pm(8.8)$ & 0.56 \\
\hline Sc II & 4314.083 & 0.62 & -0.096 & $(93.2) \pm(7.0)$ & 0.32 & $(91.4) \pm(7.9)$ & 0.33 \\
\hline Sc II & 4400.389 & 0.61 & -0.536 & $(73.8) \pm(6.3)$ & 0.48 & $(67.6) \pm(5.9)$ & 0.36 \\
\hline Sc II & 4415.557 & 0.60 & -0.668 & $(82.7) \pm(7.7)$ & 0.50 & $(78.6) \pm(8.4)$ & 0.42 \\
\hline Sc II & 5031.021 & 1.36 & -0.400 & $(31.5) \pm(3.4)$ & 0.20 & $(28.1) \pm(6.2)$ & 0.27 \\
\hline Sc II & 5526.790 & 1.77 & 0.024 & $(28.8) \pm(3.3)$ & 0.21 & $(28.2) \pm(3.1)$ & 0.18 \\
\hline Sc II & 5657.896 & 1.51 & -0.603 & $(25.2) \pm(2.7)$ & 0.57 & $(25.3) \pm(2.3)$ & 0.49 \\
\hline Ti I & 3989.758 & 0.02 & -0.130 & $65.8 \pm 6.3$ & 2.04 & - \pm- & - \\
\hline Ti I & 3998.636 & 0.05 & 0.020 & $73.1 \pm 10.3$ & 2.10 & $72.6 \pm 8.2$ & 1.97 \\
\hline Ti I & 4981.730 & 0.85 & 70 & \pm 4.8 & 2. & $59.3 \pm 5.4$ & 1.99 \\
\hline Ti I & 4991.066 & 0.84 & 0.450 & \pm 5.4 & 1.97 & $51.9 \pm 3.5$ & 1.97 \\
\hline Ti I & 4999.503 & 0.83 & 0.320 & $.7 \pm 3.7$ & 1.91 & $41.9 \pm 3.5$ & 1.92 \\
\hline Ti I & 5014.276 & 0.81 & 0.040 & $34.6 \pm 4.2$ & 2.12 & $43.2 \pm 5.7$ & 2.20 \\
\hline Ti I & 5039.958 & 0.02 & -1.080 & $26.7 \pm 2.6$ & 2.06 & $26.7 \pm 3.4$ & 1.98 \\
\hline Ti I & 5064.653 & 0.05 & -0.940 & $30.1 \pm 2.9$ & 2.02 & $29.5 \pm 2.7$ & 1.93 \\
\hline Ti I & 5173.743 & 0.00 & -1.060 & $29.7 \pm 2.9$ & 2.06 & $31.9 \pm 3.2$ & 2.03 \\
\hline Ti I & 5192.969 & 0.02 & -0.950 & $33.7 \pm 2.6$ & 2.06 & $25.7 \pm 3.0$ & 1.81 \\
\hline Ti I & 5210.384 & 0.05 & -0.820 & $37.5 \pm 3.2$ & 2.03 & $38.1 \pm 3.0$ & 1.96 \\
\hline Ti II & 3913.461 & 1.12 & -0.360 & $111.3 \pm 11.0$ & 2.23 & $130.4 \pm 10.5$ & 2.54 \\
\hline Ti II & 4028.338 & 1.89 & -0.920 & $51.8 \pm 5.3$ & 2.27 & - \pm- & - \\
\hline Ti II & 4290.215 & 1.16 & -0.870 & $101.5 \pm 10.9$ & 2.37 & $96.1 \pm 9.2$ & 2.16 \\
\hline Ti II & 4337.914 & 1.08 & -0.960 & - \pm- & - & $88.6 \pm 9.9$ & 1.96 \\
\hline Ti II & 4394.059 & 1.22 & -1.770 & $57.1 \pm 6.0$ & 2.31 & $55.2 \pm 5.1$ & 2.24 \\
\hline Ti II & 4395.031 & 1.08 & -0.540 & $119.8 \pm 11.3$ & 2.34 & $120.5 \pm 9.0$ & 2.26 \\
\hline Ti II & 4395.839 & 1.24 & -1.930 & $55.9 \pm 5.7$ & 2.48 & $46.0 \pm 5.4$ & 2.26 \\
\hline Ti II & 4399.765 & 1.24 & -1.200 & $89.2 \pm 8.7$ & 2.46 & $89.0 \pm 8.7$ & 2.38 \\
\hline Ti II & 4417.713 & 1.1 & -1.190 & $96.6 \pm 8.3$ & 2.54 & $97.1 \pm 8.8$ & 2.47 \\
\hline Ti II & 4443.801 & 1.08 & -0.710 & $103.7 \pm 8.1$ & 2.11 & $112.6 \pm 7.4$ & 2.23 \\
\hline Ti II & 4444.554 & 1.12 & -2.200 & - \pm- & - & $44.5 \pm 4.8$ & 2.34 \\
\hline Ti II & 4450.482 & 1.08 & -1.520 & $82.1 \pm 7.5$ & 2.41 & $80.9 \pm 8.8$ & 2.32 \\
\hline Ti II & 4464.449 & 1.16 & -1.810 & - \pm- & - & $59.0 \pm 7.2$ & 2.27 \\
\hline Ti II & 4468.493 & 1.13 & -0.630 & $95.2 \pm 7.3$ & 1.89 & - \pm- & - \\
\hline Ti II & 4501.270 & 1.12 & -0.770 & $116.2 \pm 12.6$ & 2.48 & - \pm- & - \\
\hline Ti II & 4865.610 & 1.12 & -2.700 & - \pm- & - & $25.9 \pm 3.8$ & 2.43 \\
\hline
\end{tabular}

Notes. Fe I lines marked with * were not used for the mean Fe I abundance determination as their $\chi_{\text {ex }}$ is lower than 1.4, their EW is too large or too small, as explained in Sect. 3.4.
Table A.1. continued.

\begin{tabular}{|c|c|c|c|c|c|c|c|}
\hline \multirow{2}{*}{$\begin{array}{l}\text { El. } \\
\text { Ti II }\end{array}$} & \multirow{2}{*}{ 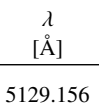 } & \multirow{2}{*}{$\begin{array}{l}\begin{array}{c}\chi_{\mathrm{ex}} \\
{[\mathrm{eV}]}\end{array} \\
1.89\end{array}$} & \multirow{2}{*}{$\begin{array}{l}\log (g f) \\
-1.340\end{array}$} & \multicolumn{2}{|c|}{$\begin{array}{c}\mathrm{EW}[\mathrm{mA}] \quad \log \epsilon(\mathrm{X}) \\
\mathrm{S} 04-130\end{array}$} & \multicolumn{2}{|c|}{$\begin{array}{c}\mathrm{EW}[\mathrm{mA}] \\
\mathrm{S} 11-97\end{array}$} \\
\hline & & & & $36.0 \pm 4.0$ & 2.25 & $34.0 \pm 2.9$ & 2.20 \\
\hline Ti III & 5154.068 & 1.57 & -1.750 & $31.4 \pm 2.6$ & 2.16 & $35.5 \pm 3.3$ & 2.22 \\
\hline Ti II & 5185.902 & 1.89 & -1.410 & $33.1 \pm 3.4$ & 2.26 & $29.8 \pm 2.8$ & 2.18 \\
\hline Ti II & 5188.687 & 1.58 & -1.050 & $77.5 \pm 7.0$ & 2.31 & $73.7 \pm 6.0$ & 2.20 \\
\hline Ti II & 5226.539 & 1.57 & -1.260 & $64.4 \pm 5.3$ & 2.25 & $62.6 \pm 4.9$ & 2.19 \\
\hline Ti II & 5336.786 & 1.58 & -1.600 & $48.0 \pm 3.9$ & 2.31 & $42.1 \pm 3.8$ & 2.19 \\
\hline Ti II & 5381.021 & 1.57 & -1.970 & $30.8 \pm 3.0$ & 2.35 & $33.3 \pm 2.9$ & 2.38 \\
\hline $\mathrm{V}_{\text {III }}$ & 3951.957 & 1.48 & -0.730 & $(34.4) \pm(4.8)$ & 0.95 & - \pm- & - \\
\hline CrI & 4254.352 & 0.00 & -0.090 & - \pm- & - & $(119.5) \pm(8.9)$ & 2.65 \\
\hline CrI & 4274.812 & 0.00 & -0.220 & - \pm- & - & $(121.3) \pm(10.1)$ & 2.68 \\
\hline CrI & 4289.730 & 0.00 & -0.370 & $(96.8) \pm(8.0)$ & 2.53 & $(113.9) \pm(9.9)$ & 2.81 \\
\hline CrI & 5206.023 & 0.94 & 0.020 & $(82.8) \pm(5.7)$ & 2.46 & $(80.9) \pm(5.5)$ & 2.33 \\
\hline $\mathrm{CrI}$ & 5208.409 & 0.94 & 0.170 & $(64.9) \pm(26.3)$ & 2.46 & $(106.1) \pm(11.3)$ & 2.33 \\
\hline CrI & 5296.691 & 0.98 & -1.360 & - \pm- & - & $(16.8) \pm(1.9)$ & 2.48 \\
\hline CrI & 5298.271 & 0.98 & -1.140 & $(29.6) \pm(2.8)$ & 2.61 & $(27.3) \pm(2.4)$ & 2.48 \\
\hline CrI & 5345.796 & 1.00 & -0.896 & $(36.3) \pm(3.3)$ & 2.60 & $(32.7) \pm(3.0)$ & 2.48 \\
\hline CrI & 5348.314 & 1.00 & -1.210 & $(22.3) \pm(2.1)$ & 2.58 & $(21.1) \pm(2.5)$ & 2.47 \\
\hline $\mathrm{CrI}$ & 5409.784 & 1.03 & -0.670 & $(47.3) \pm(4.7)$ & 2.48 & - \pm- & - \\
\hline Mn I & 4030.750 & 0.00 & -0.494 & $(142.7) \pm(12.1)$ & 2.23 & $(127.8) \pm(11.9)$ & 2.07 \\
\hline Mn I & 4033.060 & 0.00 & -0.644 & $(122.2) \pm(11.2)$ & 2.22 & $(130.0) \pm(15.2)$ & 2.06 \\
\hline Mn I & 4034.480 & 0.00 & -0.842 & $(132.2) \pm(9.9)$ & 2.22 & $(94.8) \pm(10.8)$ & 2.08 \\
\hline Mn I & 4041.350 & 2.11 & 0.277 & $(38.2) \pm(5.6)$ & 2.22 & $(47.1) \pm(5.9)$ & 2.07 \\
\hline Mn I & 4823.520 & 2.32 & 0.121 & $(25.2) \pm(2.5)$ & 2.16 & $(27.3) \pm(3.3)$ & 2.06 \\
\hline $\mathrm{Fe} I$ & 4859.741 & 2.88 & -0.764 & $59.4 \pm 5.0$ & 4.56 & - \pm- & - \\
\hline $\mathrm{Fe} I$ & 4871.318 & 2.87 & -0.363 & $73.3 \pm 5.5$ & 4.43 & $74.8 \pm 6.4$ & 4.36 \\
\hline $\mathrm{Fe} I$ & 4872.138 & 2.88 & -0.567 & $58.8 \pm 4.7$ & 4.36 & $61.1 \pm 5.8$ & 4.33 \\
\hline $\mathrm{Fe} I$ & 4890.755 & 2.88 & -0.394 & $78.2 \pm 6.1$ & 4.57 & $75.4 \pm 5.6$ & 4.42 \\
\hline $\mathrm{Fe} I$ & 4891.492 & 2.85 & -0.112 & $83.3 \pm 6.4$ & 4.37 & $94.0 \pm 7.4$ & 4.48 \\
\hline $\mathrm{Fe} I$ & 4903.310 & 2.88 & -0.926 & $52.6 \pm 4.3$ & 4.60 & $43.6 \pm 3.5$ & 4.37 \\
\hline $\mathrm{Fe} I$ & 4918.994 & 2.87 & -0.342 & $78.0 \pm 6.3$ & 4.50 & $77.8 \pm 6.0$ & 4.39 \\
\hline $\mathrm{Fe} I$ & 4920.502 & 2.83 & 0.068 & $93.5 \pm 7.9$ & 4.38 & .5 & 4.33 \\
\hline $\mathrm{Fe} I$ & 4924.770 & 2.28 & -2.241 & - \pm- & - & $28.4 \pm 3.0$ & 4.63 \\
\hline $\mathrm{Fe} I$ & 4938.814 & 2.88 & -1.077 & $32.7 \pm 4.2$ & 4.36 & $46.4 \pm 4.4$ & 4.56 \\
\hline $\mathrm{Fe} I$ & 4939.687 & 0.86 & -3.340 & - \pm- & - & $76.0 \pm 6.2$ & $4.74 *$ \\
\hline $\mathrm{Fe} I$ & 4994.129 & 0.92 & -3.080 & $63.0 \pm 18.6$ & $4.42 *$ & - \pm- & - \\
\hline $\mathrm{Fe} I$ & 5006.119 & 2.83 & -0.638 & $71.0 \pm 6.0$ & 4.59 & $72.6 \pm 4.7$ & 4.53 \\
\hline $\mathrm{Fe} I$ & 5041.072 & 0.96 & -3.087 & $53.3 \pm 19.7$ & $4.30^{*}$ & - \pm- & - \\
\hline $\mathrm{Fe} I$ & 5041.756 & 1.49 & -2.203 & $82.1 \pm 7.0$ & 4.66 & $89.3 \pm 6.5$ & 4.68 \\
\hline $\mathrm{Fe} I$ & 5049.820 & 2.28 & -1.355 & $67.8 \pm 5.1$ & 4.54 & $69.1 \pm 4.6$ & 4.47 \\
\hline $\mathrm{Fe}_{\mathrm{I}}$ & 5051.634 & 0.92 & -2.795 & $107.4 \pm 8.6$ & $5.03^{*}$ & $108.4 \pm 8.5$ & $4.88^{*}$ \\
\hline Fe I & 5068.766 & 2.94 & -1.042 & - \pm- & - & $37.3 \pm 3.7$ & 4.43 \\
\hline $\mathrm{Fe} I$ & 5079.223 & 2.20 & -2.067 & $43.3 \pm 3.7$ & 4.69 & - \pm- & - \\
\hline $\mathrm{Fe} I$ & 5079.740 & 0.99 & -3.220 & $76.0 \pm 6.1$ & $4.89^{*}$ & - \pm- & - \\
\hline $\mathrm{Fe} I$ & 5083.338 & 0.96 & -2.958 & $88.7 \pm 6.9$ & $4.84 *$ & $91.2 \pm 7.2$ & $4.76^{*}$ \\
\hline $\mathrm{Fe} I$ & 5110.413 & 0.00 & -3.760 & $116.9 \pm 7.7$ & $4.91 *$ & $117.3 \pm 8.1$ & $4.73^{*}$ \\
\hline $\mathrm{Fe}_{\mathrm{I}}$ & 5123.720 & 1.01 & -3.068 & $81.7 \pm 5.8$ & $4.87^{*}$ & $77.6 \pm 5.5$ & $4.67 *$ \\
\hline $\mathrm{Fe} I$ & 5127.359 & 0.92 & -3.307 & $78.6 \pm 5.2$ & $4.92 *$ & $68.7 \pm 5.0$ & $4.62 *$ \\
\hline $\mathrm{Fe} I$ & 5131.468 & 2.22 & -2.515 & $21.1 \pm 1.7$ & 4.70 & - \pm- & - \\
\hline $\mathrm{Fe} I$ & 5141.739 & 2.42 & -1.964 & $23.9 \pm 2.6$ & 4.47 & - \pm- & - \\
\hline $\mathrm{Fe} I$ & 5150.839 & 0.99 & -3.003 & $75.2 \pm 6.1$ & $4.64 *$ & $74.4 \pm 5.8$ & $4.51^{*}$ \\
\hline $\mathrm{Fe} I$ & 5151.911 & 1.01 & -3.322 & $63.1 \pm 4.9$ & $4.76^{*}$ & $63.1 \pm 5.0$ & $4.66^{*}$ \\
\hline $\mathrm{Fe} I$ & 5166.282 & 0.00 & -4.195 & $97.1 \pm 7.6$ & $4.93 *$ & $91.2 \pm 8.2$ & $4.66^{*}$ \\
\hline $\mathrm{Fe} I$ & 5171.596 & 1.49 & -1.793 & & - & $108.0 \pm 7.2$ & 4.62 \\
\hline $\mathrm{Fe} I$ & 5191.455 & 3.04 & -0.551 & $58.9 \pm 4.8$ & 4.50 & $56.4 \pm 3.9$ & 4.39 \\
\hline $\mathrm{Fe} I$ & 5192.344 & 3.00 & -0.421 & - \pm- & - & $62.7 \pm 5.4$ & 4.32 \\
\hline $\mathrm{Fe} I$ & 5194.941 & 1.56 & -2.090 & $81.6 \pm 6.2$ & 4.60 & $91.7 \pm 5.7$ & 4.68 \\
\hline $\mathrm{Fe} I$ & 5198.711 & 2.22 & -2.135 & $37.1 \pm 3.5$ & 4.66 & - \pm- & - \\
\hline $\mathrm{Fe} I$ & 5202.336 & 2.18 & -1.838 & $60.3 \pm 4.5$ & 4.72 & $56.5 \pm 4.9$ & 4.57 \\
\hline $\mathrm{Fe} I$ & 5216.274 & 1.61 & -2.150 & $84.6 \pm 5.8$ & 4.78 & $83.7 \pm 6.9$ & 4.64 \\
\hline $\mathrm{Fe} I$ & 5217.389 & 3.21 & -1.070 & $29.3 \pm 2.6$ & 4.68 & $21.9 \pm 1.9$ & 4.46 \\
\hline $\mathrm{Fe} I$ & 5225.526 & 0.11 & -4.789 & $54.2 \pm 4.7$ & $4.88^{*}$ & $55.5 \pm 4.2$ & $4.79 *$ \\
\hline $\mathrm{Fe} I$ & 5232.940 & 2.94 & -0.058 & - \pm- & - & $93.5 \pm 7.0$ & 4.46 \\
\hline $\mathrm{Fe} I$ & 5254.956 & 0.11 & -4.764 & $57.2 \pm 4.7$ & $4.90^{*}$ & $55.7 \pm 4.9$ & $4.77 *$ \\
\hline $\mathrm{Fe} I$ & 5266.555 & 3.00 & -0.386 & $70.4 \pm 4.7$ & 4.50 & $63.8 \pm 5.0$ & 4.29 \\
\hline $\mathrm{Fe} I$ & 5269.537 & 0.86 & -1.321 & $168.6 \pm 13.4$ & $4.60^{*}$ & $162.3 \pm 12.9$ & $4.29 *$ \\
\hline $\mathrm{Fe} I$ & 5281.790 & 3.04 & -0.834 & $45.6 \pm 3.5$ & 4.53 & - \pm- & - \\
\hline $\mathrm{Fe} I$ & 5302.300 & 3.28 & -0.720 & $40.6 \pm 3.8$ & 4.63 & $33.0 \pm 2.5$ & 4.43 \\
\hline $\mathrm{Fe} I$ & 5307.361 & 1.61 & -2.987 & - \pm- & - & $36.5 \pm 3.1$ & 4.64 \\
\hline $\mathrm{Fe} I$ & 5324.179 & 3.21 & -0.103 & $67.9 \pm 5.5$ & 4.43 & $69.3 \pm 5.8$ & 4.37 \\
\hline $\mathrm{Fe} I$ & 5328.039 & 0.92 & -1.466 & $157.6 \pm 12.2$ & $4.60^{*}$ & $165.1 \pm 12.8$ & $4.53 *$ \\
\hline
\end{tabular}


Table A.1. continued.

\begin{tabular}{|c|c|c|c|c|c|c|c|}
\hline \multirow{2}{*}{$\begin{array}{l}\text { El. } \\
\text { Fe I }\end{array}$} & \multirow{2}{*}{$\begin{array}{c}\lambda \\
{[\AA]} \\
5332.899\end{array}$} & \multirow{2}{*}{$\begin{array}{l}\begin{array}{l}\chi_{\mathrm{ex}} \\
{[\mathrm{eV}]}\end{array} \\
1.56\end{array}$} & \multirow{2}{*}{$\begin{array}{l}\log (g f) \\
-2.777\end{array}$} & \multicolumn{2}{|c|}{$\begin{array}{c}\mathrm{EW}[\mathrm{mA}] \quad \log \epsilon(\mathrm{X}) \\
\mathrm{S} 04-130\end{array}$} & \multicolumn{2}{|c|}{$\begin{array}{c}\mathrm{EW}[\mathrm{mA}] \\
\mathrm{S} 11-97\end{array}$} \\
\hline & & & & $51.7 \pm 4.5$ & 4.70 & $44.6 \pm 3.7$ & 4.50 \\
\hline $\mathrm{Fe}_{\mathrm{I}}$ & 5367.466 & 4.41 & 0.443 & $20.4 \pm 2.2$ & 4.42 & - \pm- & - \\
\hline $\mathrm{Fe} I$ & 5369.961 & 4.37 & 0.536 & $31.5 \pm 3.3$ & 4.54 & $30.3 \pm 2.7$ & 4.47 \\
\hline $\mathrm{Fe} I$ & 5371.489 & 0.96 & -1.645 & $152.3 \pm 11.0$ & $4.72 *$ & $148.0 \pm 11.2$ & $4.44^{*}$ \\
\hline $\mathrm{Fe} I$ & 5383.369 & 4.31 & 0.645 & $37.2 \pm 2.6$ & 4.47 & - \pm- & - \\
\hline $\mathrm{Fe} I$ & 5393.167 & 3.24 & -0.715 & $36.3 \pm 3.1$ & 4.49 & - \pm- & - \\
\hline $\mathrm{Fe} I$ & 5397.128 & 0.92 & -1.993 & $137.9 \pm 9.3$ & $4.72 *$ & $140.7 \pm 9.6$ & $4.59^{*}$ \\
\hline $\mathrm{Fe} I$ & 5405.774 & 0.99 & -1.844 & $143.0 \pm 10.3$ & $4.77^{*}$ & $144.3 \pm 9.4$ & $4.60^{*}$ \\
\hline $\mathrm{Fe} I$ & 5410.910 & 4.47 & 0.398 & - \pm- & - & $22.3 \pm 2.4$ & 4.55 \\
\hline $\mathrm{Fe} I$ & 5424.068 & 4.32 & 0.520 & - \pm- & - & $34.1 \pm 3.1$ & 4.50 \\
\hline $\mathrm{Fe} I$ & 5429.696 & 0.96 & -1.879 & $149.9 \pm 10.7$ & $4.88^{*}$ & $148.3 \pm 11.1$ & $4.66^{*}$ \\
\hline $\mathrm{Fe} I$ & 5434.523 & 1.01 & -2.122 & $129.9 \pm 9.3$ & $4.81^{*}$ & $127.5 \pm 9.2$ & $4.59^{*}$ \\
\hline $\mathrm{Fe} I$ & 5446.917 & 0.99 & -1.914 & $140.1 \pm 10.2$ & $4.77^{*}$ & $139.6 \pm 11.1$ & $4.57^{*}$ \\
\hline $\mathrm{Fe} I$ & 5455.609 & 1.01 & -2.091 & $132.9 \pm 11.7$ & $4.83^{*}$ & $140.9 \pm 12.0$ & $4.80^{*}$ \\
\hline $\mathrm{Fe} I$ & 5497.516 & 1.01 & -2.849 & $96.5 \pm 6.9$ & $4.86^{*}$ & $99.8 \pm 7.7$ & $4.78^{*}$ \\
\hline Fe I & 5501.465 & 0.96 & -3.047 & $93.4 \pm 6.9$ & $4.93^{*}$ & $94.6 \pm 5.8$ & $4.81^{*}$ \\
\hline $\mathrm{Fe}_{\mathrm{I}}$ & 5506.779 & 0.99 & -2.797 & $110.7 \pm 7.2$ & $5.06^{*}$ & $106.8 \pm 7.6$ & $4.83^{*}$ \\
\hline $\mathrm{Fe} I$ & 5569.618 & 3.42 & -0.486 & $41.6 \pm 3.2$ & 4.56 & $35.2 \pm 3.4$ & 4.39 \\
\hline $\mathrm{Fe} I$ & 5572.842 & 3.40 & -0.275 & - \pm- & - & $49.2 \pm 3.5$ & 4.40 \\
\hline $\mathrm{Fe}_{\mathrm{I}}$ & 5586.755 & 3.37 & -0.120 & $63.5 \pm 5.1$ & 4.53 & $59.9 \pm 4.6$ & 4.39 \\
\hline Fe I & 5615.644 & 3.33 & 0.050 & - \pm- & - & $70.6 \pm 5.5$ & 4.36 \\
\hline $\mathrm{Fe} I$ & 6065.482 & 2.61 & -1.530 & $44.7 \pm 3.2$ & 4.61 & $43.6 \pm 3.3$ & 4.52 \\
\hline $\mathrm{Fe}_{\mathrm{I}}$ & 6136.615 & 2.45 & -1.400 & $62.4 \pm 4.1$ & 4.57 & $66.5 \pm 4.7$ & 4.56 \\
\hline $\mathrm{Fe} I$ & 6137.691 & 2.59 & -1.403 & $51.0 \pm 3.7$ & 4.55 & $56.4 \pm 3.9$ & 4.57 \\
\hline $\mathrm{Fe} I$ & 6191.558 & 2.43 & -1.417 & - \pm- & - & $42.0 \pm 25.8$ & $4.15^{*}$ \\
\hline $\mathrm{Fe} I$ & 6213.429 & 2.22 & -2.482 & $25.2 \pm 2.5$ & 4.68 & - \pm- & - \\
\hline $\mathrm{Fe} I$ & 6246.318 & 3.60 & -0.733 & $20.6 \pm 1.9$ & 4.55 & - \pm- & - \\
\hline $\mathrm{Fe} I$ & 6252.555 & 2.40 & -1.687 & $51.7 \pm 4.2$ & 4.61 & $54.6 \pm 3.8$ & 4.58 \\
\hline $\mathrm{Fe} I$ & 6335.330 & 2.20 & -2.177 & $40.5 \pm 3.3$ & 4.63 & - \pm- & - \\
\hline $\mathrm{Fe} I$ & 6393.601 & 2.43 & -1.432 & $57.9 \pm 4.7$ & 4.48 & $60.4 \pm 4.7$ & 4.44 \\
\hline $\mathrm{Fe} I$ & 6411.648 & 3.65 & -0.595 & - \pm- & - & $24.5 \pm 2.1$ & 4.52 \\
\hline Fe I & 6421.350 & 2.28 & -2.027 & - \pm- & - & $47.1 \pm 3.4$ & 4.62 \\
\hline $\mathrm{Fe} I$ & 6430.845 & 2.18 & -2.006 & - \pm- & - & $57.4 \pm 4.2$ & 4.63 \\
\hline $\mathrm{Fe} I$ & 6494.980 & 2.40 & -1.273 & $82.4 \pm 6.5$ & 4.69 & $82.9 \pm 5.5$ & 4.60 \\
\hline $\mathrm{Fe} I$ & 6592.913 & 2.73 & -1.473 & - \pm- & - & $37.1 \pm 2.9$ & 4.46 \\
\hline $\mathrm{Fe}_{\mathrm{I}}$ & 6677.985 & 2.69 & -1.418 & $52.0 \pm 3.8$ & 4.67 & $51.9 \pm 3.9$ & 4.60 \\
\hline Fe II & 4923.921 & 2.89 & -1.320 & $105.3 \pm 8.4$ & 4.84 & $77.4 \pm 20.4$ & 4.19 \\
\hline $\mathrm{Fe}$ II & 5018.436 & 2.89 & -1.220 & $112.8 \pm 9.0$ & 4.87 & $82.2 \pm 18.1$ & 4.17 \\
\hline $\mathrm{Fe}$ II & 5197.567 & 3.23 & -2.100 & - \pm- & - & $32.9 \pm 3.0$ & 4.52 \\
\hline $\mathrm{Fe}$ II & 5234.623 & 3.22 & -2.230 & $42.2 \pm 3.1$ & 4.82 & - \pm- & - \\
\hline $\mathrm{Fe}$ II & 5275.997 & 3.20 & -1.940 & $50.1 \pm 4.8$ & 4.65 & $54.6 \pm 5.0$ & 4.72 \\
\hline Fe II & 5284.103 & 2.89 & -2.990 & $24.3 \pm 2.3$ & 4.80 & - \pm- & - \\
\hline CoI & 3845.468 & 0.92 & 0.010 & $(71.7) \pm(7.0)$ & 2.12 & $(76.5) \pm(8.0)$ & 1.75 \\
\hline CoI & 3894.077 & 1.05 & 0.100 & $(95.1) \pm(10.0)$ & 2.19 & $(105.7) \pm(11.0)$ & 1.74 \\
\hline CoI & 3995.307 & 0.92 & -0.220 & $(73.3) \pm(7.9)$ & 1.92 & $(80.2) \pm(6.0)$ & 1.80 \\
\hline Co I & 4121.318 & 0.92 & -0.320 & $(92.0) \pm(8.2)$ & 1.96 & $(76.7) \pm(6.6)$ & 1.82 \\
\hline Ni I & 3858.297 & 0.42 & -0.960 & $(116.3) \pm(9.3)$ & 3.44 & - \pm- & - \\
\hline Ni I & 5084.096 & 3.68 & 0.030 & - \pm- & - & $(11.4) \pm(2.1)$ & 3.34 \\
\hline Ni I & 5155.764 & 3.90 & 0.074 & - \pm- & - & $(10.4) \pm(1.5)$ & 3.46 \\
\hline Ni I & 5476.904 & 1.83 & -0.780 & $(55.9) \pm(18.2)$ & 3.21 & $(76.2) \pm(5.5)$ & 3.13 \\
\hline Ni I & 6643.630 & 1.68 & -2.220 & $(20.7) \pm(2.0)$ & 3.33 & - \pm- & - \\
\hline Ni I & 6767.772 & 1.83 & -2.140 & $(24.9) \pm(2.6)$ & 3.60 & $(19.1) \pm(3.2)$ & 3.54 \\
\hline $\mathrm{Zn} \mathrm{I}$ & 4810.528 & 4.08 & -0.137 & $(29.1) \pm(3.7)$ & 2.25 & $(23.0) \pm(2.9)$ & 2.10 \\
\hline Sr II & 4077.709 & 0.00 & 0.167 & $(144.5) \pm(12.0)$ & 0.17 & $(81.6) \pm(15.4)$ & -0.60 \\
\hline Sr II & 4215.519 & 0.00 & -0.145 & $(122.0) \pm(9.6)$ & -0.02 & $(132.1) \pm(13.5)$ & -0.35 \\
\hline $\mathrm{Y}_{\mathrm{II}}$ & 4883.682 & 1.08 & 0.070 & $(13.2) \pm(2.0)$ & -1.20 & $(14.9) \pm(2.4)$ & -1.33 \\
\hline $\mathrm{Y}_{\text {II }}$ & 5200.410 & 0.99 & -0.570 & - \pm- & - & $(12.0) \pm(1.5)$ & -1.01 \\
\hline $\mathrm{Y}_{\text {II }}$ & 5205.722 & 1.03 & -0.340 & $(11.7) \pm(2.1)$ & -1.12 & - \pm- & - \\
\hline Zr II & 4208.980 & 0.71 & -0.510 & $(24.2) \pm(3.6)$ & -0.25 & $(30.1) \pm(3.1)$ & -0.39 \\
\hline Ba II & 4934.076 & 0.00 & -0.150 & $(89.6) \pm(7.4)$ & -1.59 & $(92.6) \pm(8.4)$ & -1.62 \\
\hline Ba II & 5853.668 & 0.60 & -1.000 & $(13.3) \pm(1.4)$ & -1.46 & - \pm- & - \\
\hline Ba II & 6141.713 & 0.70 & -0.076 & $(42.1) \pm(4.0)$ & -1.64 & $(44.8) \pm(3.3)$ & -1.65 \\
\hline Ba II & 6496.897 & 0.60 & -0.377 & $(39.2) \pm(3.3)$ & -1.53 & $(38.4) \pm(3.4)$ & -1.57 \\
\hline Pr II & 4143.112 & 0.37 & 0.609 & - \pm- & - & $14.6 \pm 4.3$ & -1.82 \\
\hline Nd II & 4446.380 & 0.20 & -0.350 & $15.1 \pm 4.5$ & -1.12 & - \pm- & - \\
\hline
\end{tabular}

